\title{
Spontaneous Emission of Spiral Inertia-Gravity Waves and Formation of Elliptical Eyewalls in Tropical Cyclone-Like Vortices: Three-Dimensional Nonlinear Simulations
}

\author{
Konstantinos MenelaOU AND M. K. YaU \\ Department of Atmospheric and Oceanic Sciences, McGill University, Montreal, Quebec, Canada
}

(Manuscript received 15 November 2017, in final form 24 April 2018)

\begin{abstract}
Although intense tropical cyclones (TCs) are considered to be axisymmetric vortices, observations reveal that they are often highly asymmetric. Better understanding of the underlying asymmetric dynamics is a critical step toward advancing TC intensity forecasting. In this paper, we revisit the mechanisms behind one of the most frequent asymmetric patterns: the deformation of the core into an elliptical shape. Previously, elliptical eyewalls were primarily thought to be an outcome of barotropic instability, a mechanism that involves the coupling and mutual growth of counterpropagating vortex Rossby (VR) waves. These results were largely based on simplified numerical models that filter out inertia-gravity (IG) waves. Consideration of IG waves introduces the possibility of an additional instability mechanism, one that involves a VR wave that spontaneously emits a spiral IG wave into the environment. We provide evidence that elliptical eyewalls, which may form within a three-dimensional primitive-equation nonlinear model that supports both instability types, can solely originate by the mechanism of spontaneous radiative imbalance. These evidences are supported by a number of nonlinear simulations, supplemental linear eigenmode analysis, and a linear simulation. The potential role of a multimechanistic instability is also briefly addressed.
\end{abstract}

\section{Introduction}

There have been many attempts to understand how internal dynamics regulate the intensity of a tropical cyclone (TC). Most of these efforts were based on theoretical and numerical studies that neglect axial asymmetry. Since observations frequently show that TCs can be highly asymmetric during their intensification phase, such an approximation is not always justified. Asymmetries are more than geometric curiosities, and in fact, the current insufficient understanding of their dynamics is considered one main reason behind the relatively slow improvement in the accurate prediction of TC intensity over the past few decades (e.g., Wang and $\mathrm{Wu} 2004)$. This paper is an effort to provide further insight behind the instability mechanisms that can give rise to such TC asymmetries.

The definition of TC asymmetries is here reserved for the deformation of the vortex core into a variety of shapes, including vortex misalignments, ellipses, triangles, and polygons. Elliptical shapes and misalignments

Corresponding author: Konstantinos Menelaou, konstantinos. menelaou@mail.mcgill.ca are perhaps among the most commonly observed. In particular, Li et al. (2013) analyzed 83 TC cases from synthetic aperture radar (SAR) imagery and found that a majority of them exhibited core asymmetries of these two types. At the mature TC stage, pronounced elliptical asymmetries were captured by both land-based radar (Mitsuta and Yoshizumi 1973; Kuo et al. 1999; Corbosiero et al. 2006) and airborne dual-Doppler radar measurements (Reasor et al. 2000; Aberson et al. 2006). Likewise, these asymmetries were also seen in cloudresolving simulations (e.g., Braun 2002; Nuissier et al. 2005). Echoing Braun (2002), the distribution of radial flow, vertical motion, and precipitation were strongly modulated by the pronounced elliptical asymmetry. It is reasonable to ask what mechanisms can generate these elliptical asymmetries.

Inferring causality from either radar imagery or cloudresolving simulations alone is not straightforward since the former provides only limited information and the information from the latter might be too complex. Actually, most of what is currently known relies on knowledge gained from simplified models, including two-dimensional barotropic nondivergent models (Reasor et al. 2000; Kossin et al. 2000) and the asymmetric balance 
(AB) model $^{1}$ (Oda et al. 2005). According to Reasor et al. (2000), elliptical eyewalls can be the outcome of barotropic instability across the eyewall. They verified this instability by first examining the symmetric radial vorticity profile of Hurricane Olivia (derived from the dual-Doppler radar winds) and recognizing its resemblance to an annular vortex ring. It is known that a pronounced peak in the radial profile of vertical vorticity may accommodate counterpropagating vortex Rossby (VR) waves with the ability to phase lock and induce mutual growth. Then they revealed from linear stability analysis the existence of unstable modes. A nonlinear simulation further verified the release of this instability. On the other hand, Kossin et al. (2000) propose an alternative instability mechanism that requires the additional presence of a secondary ring of elevated vorticity (intended to mimic a concentric eyewall pattern). In this subtle variant of the typical barotropic instability (which they referred to as type-2 instability), elliptical eyewalls are the result of the unstable interaction between the VR wave excited on the outer edge of the primary eyewall and the VR wave excited on the inner edge of the secondary eyewall. Similarly, the threedimensional AB stability analysis of Oda et al. (2005) also supported a type- 2 instability as the mechanism responsible for the elliptical eyewall of Typhoon Herb.

The aforementioned discussion implies that elliptical eyewalls primarily result from the unstable interaction of two VR waves. Although this is a plausible scenario, a potential caveat of the two sets of equations (nondivergent barotropic vorticity and $\mathrm{AB}$ approximation) used to infer this conclusion is that they filter out inertia-gravity (IG) waves. Adding IG waves to the system introduces the possibility of an additional sustained exponential instability, which involves a VR wave in the TC core that spontaneously emits a frequency-matched spiral IG wave into the environment (e.g., Ford 1994a,b; Plougonven and Zeitlin 2002; Schecter and Montgomery 2003, 2004; Schecter 2008; Hodyss and Nolan 2008). The IG wave radiation can feed back positively on the VR wave, causing both to grow.

Until recently, the potential role of spontaneous radiative imbalance in TCs was tacitly assumed as inconsequential. However, the comprehensive linear stability analysis conducted by Menelaou et al. (2016) illustrated that IG wave radiation can be quite fast and potentially relevant to real-world TCs. In the context of annular vortices (which support both barotropic instability and

\footnotetext{
${ }^{1}$ The theory behind the $\mathrm{AB}$ model is derived in Shapiro and Montgomery (1993).
}

spontaneous radiative imbalance), their analyses presented an extensive examination of the primary modes of instability for Rossby and Froude numbers appropriate for TCs. They showed that increasing the Froude number beyond unity tends to cause a transition (which can be either abrupt or gradual) from nonradiative instabilities (in which IG wave emissions are incidental) to radiation-driven instabilities. Abrupt transition referred to the sudden dominance of spontaneous imbalance (relative to barotropic instability), which for various case studies have been shown to occur above a critical Froude number. Gradual transition implied a continuous structural transformation of the dominant modes reflected in a diminishing influence of the interaction between two VR waves and a rise of the radiative instability mechanism. Menelaou et al. (2016) further noted (at least for their case studies) that whenever IG wave radiation predominantly controlled the instability, the dominant azimuthal wavenumber of the perturbation was primarily 2 (elliptical modes) and in some cases 3 (their Fig. 8).

Based on the above results, there is reason to believe that elliptical eyewalls may not always be the result of barotropic instability and that their generation might be due to a completely different instability mechanism. The purpose of the present study is to extend the study of Menelaou et al. (2016) to the next level of complexity toward the real atmosphere by using a threedimensional nonlinear primitive equation model. Through a series of idealized nonlinear simulations initialized with dry nonconvective annular TC-like vortices and wave activity diagnostics, as well as supplemental linear eigenmode analysis and linear simulation, it will become evident that the elliptical eyewalls, which may form within a three-dimensional nonlinear model, can originate solely from spontaneous radiative imbalance.

There are a few subtle facts worth noting before advancing to the main text. The first fact concerns an additional instability mechanism that may be potentially relevant for TCs, which involves the mutual amplification of a VR wave and the potential vorticity (PV) anomaly that it generates in a suitably conditioned critical layer (Briggs et al. 1970). The second fact concerns any feedbacks (positive or negative) that resonant stirring of $\mathrm{PV}$ in one or more critical layers may have on the growth rates of spontaneous imbalance (Schecter and Montgomery 2006). Here, we will not consider any critical layers but will report their role within nonlinear simulations in a separate paper.

The third fact concerns the possibility of a multimechanistic instability in which both barotropic instability and spontaneous radiation may be operating simultaneously (Menelaou et al. 2016). Under these 
circumstances, the role of each mechanism in destabilizing the TC is difficult to assess without the right diagnostic (Schecter and Menelaou 2017).

The fourth fact concerns our restricted use of the term spontaneous radiative imbalance. In principle, this term may refer also to a mechanism different from the one we are currently investigating, in which the presence of a VR wave can be incidental. Such instabilities involve the overreflection of an IG wave at a critical layer and the simultaneous emission of an outward-propagating IG wave into the environment (e.g., Takehiro and Hayashi 1992; Billant and Dizès 2009; Park and Billant 2013). According to Menelaou et al. (2016), such instabilities are of secondary importance in TCs.

The fifth and last fact concerns the limitations of our idealized numerical configuration. Although idealized simulations provide useful insight, when dealing with actual real TC instabilities, the influence of moisture, boundary layer processes, and vortex baroclinicity (omitted in the current setup) should also be addressed. In theory, the reduction of static stability associated with cloud coverage could significantly affect the growth (directly and indirectly) of both barotropic and radiative instabilities (Schecter and Montgomery 2007). It can also affect the excitability of a special type of azimuthal wavenumber-1 $(n=1)$ VR wave that may be responsible for the vertical misalignment of the vortex core. Using a conventional cloud model designed to include all relevant physical processes, Schecter (2015) verified in part the theory of Schecter and Montgomery (2007; which neglects any boundary layer processes and the diabatically maintained secondary circulation) that moisture acts to inhibit vortex misalignment. Despite this, Schecter (2015) also noted some discrepancies between theory and details of asymmetric convection, especially within the eyewall region of the simulated TC. In addition, he further emphasized the potential impact of symmetric secondary circulation acting independently to inhibit the vortex tilt. On the other hand, Naylor and Schecter (2014) examined moderately-highwavenumber fast barotropic instabilities ( $n$ between 4 and 6 and $e$-folding times ranging from 15 to $20 \mathrm{~min}$ ) in simulated TCs using another conventional cloud model and concluded that moisture had little impact on the early (linear) wave growth leading to the development of eyewall mesovortices. One possible explanation as to why moist secondary circulation did not have a significant impact was related to the fast time scale of the initial exponential growth, which was substantially less than the convective time scale in the eyewall. Another possible explanation was related to the location of the cloudy updraft, which was found to exist outside the region where the instability occurred. The broader applicability of this result to relatively slower elliptical modes is currently unknown and merits future investigation. Finally, with regard to the baroclinic nature of real TCs, Hodyss and Nolan (2008) noted that the inclusion of vertical shear could reduce the growth rates of radiative instabilities. However, it is interesting to note that such reduction was suggested not to be an outcome of the vertical shearing acting as a sink of perturbation kinetic energy. Instead, the smaller growth rates documented in baroclinic vortices were primarily due to an overall reduction in the radial perturbation kinetic energy production and an increase in the dissipation.

The remainder of this paper is organized as follows. Section 2 describes the configuration of the nonlinear model. Section 3 presents an overview of the control simulation. Section 4 compares the dominant asymmetries generated from the nonlinear model with a simple linear model. Section 5 presents the wave activity diagnostics. Section 6 discusses further influences on the vortex core when the initial perturbation prescribed in the control simulation takes on a different horizontal structure. Section 7 briefly describes some long-term effect of nonlinear spontaneous imbalance. Section 8 contains a description and discussion of the sensitivity experiments. Section 9 summarizes the main findings of this study. The appendix supplements the main text.

\section{Experimental setup}

\section{a. Configuration of the nonlinear model}

In this study, we make use of a subset of the Weather Research and Forecasting (WRF) Model. WRF is a state-of-the-art modeling system that is widely used for both research and operational weather forecasting. Specifically, the numerical experiments are conducted with the Advanced Research version of WRF (ARW) dynamics solver, which integrates the fully compressible, nonhydrostatic Euler equations ${ }^{2}$ (Skamarock et al. 2008). In brief, the prognostic variables of the solver include the three components of the velocity vector, potential temperature, perturbation geopotential, and perturbation surface pressure of dry air, where the perturbation quantities are defined relative to a hydrostatically balanced reference state.

The computational domain is configured on the $f$ plane with open lateral boundary conditions. The specified Coriolis parameter is $f=5 \times 10^{-5} \mathrm{~s}^{-1}$, which is

\footnotetext{
${ }^{2}$ In other words, the conventional WRF Model is integrated without including moisture and with all physics parameterizations turned off.
} 
representative of $20^{\circ} \mathrm{N}$. The fields are evolved on two nested square grids that span 720 and $3600 \mathrm{~km}$ in each orthogonal direction. The corresponding horizontal increments are 2 and $6 \mathrm{~km}$. The vertical grid mesh is the same for both grids and comprises 28 levels that extend upward to $z=20 \mathrm{~km}$, with $z$ denoting the height above mean sea level. There is no explicit horizontal and vertical diffusion in the experiments, and only the diffusion implicit in the model's odd-ordered advection schemes (fifth order here) is considered. To mitigate any artificial reflection of inertia-gravity waves from the upper boundary of the domain, an implicit upper Rayleigh damping layer (with a 5-km damping depth) for the vertical velocity is implemented.

\section{b. Setup of the control experiment}

The control experiment is designed with the main focus on the potential role of spontaneous IG wave radiation in the formation of an elliptical eyewall. The initial conditions involve a stationary axisymmetric barotropic TC-like vortex that is constructed by defining first its relative vorticity distribution $\left[\zeta(r) \equiv r^{-1} d(r v) / d r\right]$ :

$$
\zeta(r)=\zeta_{m}\left\{\frac{1}{1+\left(r / r_{v}\right)^{\Delta}}-\beta \frac{1}{1+\left[r /\left(\mu r_{v}\right)\right]^{\Delta}}\right\},
$$

in which $\left\{\zeta_{m}, r_{v}, \beta, \mu, \Delta\right\}=\left\{3.2529 \times 10^{-3} \mathrm{~s}^{-1}, 40 \mathrm{~km}\right.$, $0.3,0.5,500\}$. Here, the parameter $\zeta_{m}$ is approximately the maximum relative vorticity, $r_{v}$ represents the radius within which most of the vorticity is contained, $\beta$ controls the depth of the central vorticity deficit, $\mu$ controls the thickness of the off-center vorticity peak, and $\Delta$ controls the smoothness at the edges of the off-center vorticity peak. At this stage, we make use of a cylindrical coordinate system centered at the vortex core, and thus, $r$ denotes radius, $v$ is the azimuthal wind, and axisymmetry implies that the flow in a horizontal plane is circular. The nonmonotonic relative vorticity profile (Fig. 1a) implies that the vortex is additionally susceptible to the classic barotropic instability. The resultant maximum azimuthal velocity is $60 \mathrm{~m} \mathrm{~s}^{-1}$ and occurs at a radius of about $40 \mathrm{~km}$ (Fig. 1b). Such initial structure and magnitude resemble that of a typical well-developed TC.

The vortex is stationary because of a balance of forces, including gradient wind and hydrostatic balance:

$$
\begin{aligned}
& \frac{\partial p}{\partial r}=\rho f v+\rho \frac{v^{2}}{r}, \\
& \frac{\partial p}{\partial z}=-\rho g .
\end{aligned}
$$

Here, $p$ denotes pressure, $\rho$ is density, and $g$ is gravitational acceleration. The corresponding pressure and density fields that hold the vortex in balance are obtained following an iterative process explained in Nolan et al. (2001). In short, the far-field vertical profiles of pressure and temperature are initially prescribed according to the Jordan (1958) mean sounding for hurricane season in the West Indies. Equation (2a) is then integrated radially inward from the far field to obtain the two-dimensional $(r, z)$ pressure field. At this step, the temperature is held fixed to its current value. Equation (2b) is then applied throughout the domain to correct both the temperature and density. Finally, the previous two steps are repeated until the fields converge to a solution. Eventually, the initialization process is completed by interpolating the balanced fields onto the three-dimensional grid of the WRF Model.

To induce motion, the stationary vortex is perturbed by an asymmetric thermal pulse. The pulse is designed to be a source/sink term $Q$ in the potential temperature equation (activated only during the first $10 \mathrm{~min}$ of the simulation) instead of a more typical initial condition perturbation. This strategy is intended to mimic the diabatic processes associated with an asymmetric convective event in the vicinity of the TC core. Figures $1 \mathrm{c}$ and $1 \mathrm{~d}$ illustrate, respectively, a horizontal and vertical cross section of the pulse. Since the main focus is related to an elliptical eyewall, the pulse is prescribed in the horizontal direction to have an azimuthal wavenumber $n=2$ structure with a maximum amplitude of $5 \mathrm{~K} \mathrm{~h}^{-1}$ localized near the outer edge of the vortex core. In the vertical direction, it has a baroclinic structure with vertical wavenumber $m=2$. Similar configuration can be easily incorporated within the simple linear model, thereby allowing for a direct comparison between the two models.

\section{Overview of the control simulation}

Figure 2 shows the spatial structure of the vortex core in terms of relative vorticity at the initial time and after $12 \mathrm{~h}$ of simulation at two selected vertical levels. There are a number of important features to note. First, the initial symmetric barotropic vortex deforms into an elliptical shape. Second, the ellipticity has a baroclinic structure (phase changes in the vertical). Third, the ellipticity amplifies with time. It is the mechanism behind this elliptical amplification that we will expound in this paper.

To elaborate, it is appropriate to first investigate the evolution of the dominant azimuthal asymmetry. The following analysis makes use of an isentropic cylindrical coordinate system centered at the vortex core and the following Fourier expansion: $\alpha=\sum_{n=-\infty}^{\infty} \alpha_{n}(r, \theta, t) e^{i n \lambda}$, in which $\alpha$ is an arbitrary variable, $\lambda$ is the azimuth, and $\theta$ is potential temperature. Figure 3 (top panels) 

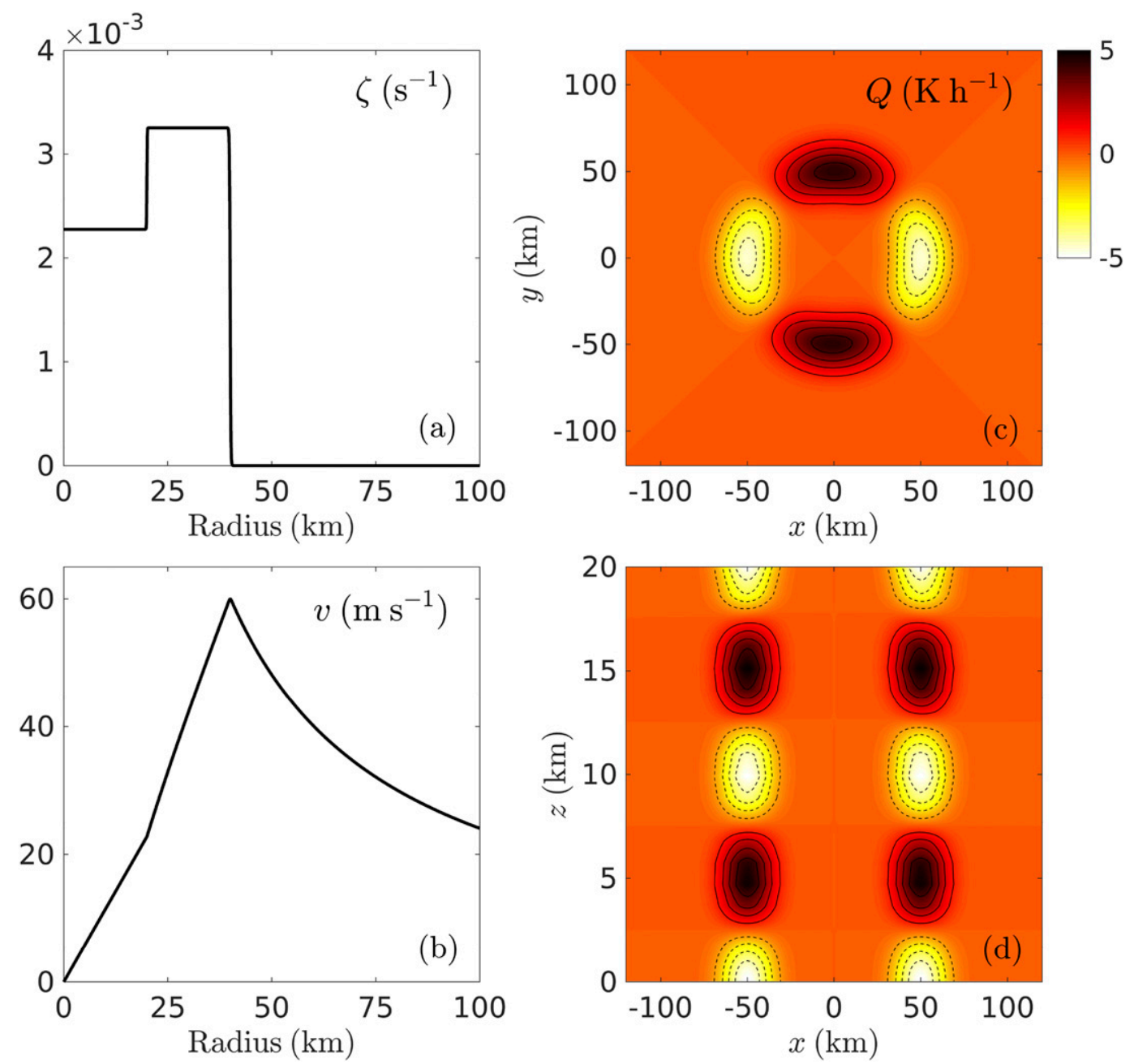

FIG. 1. Radial profile of the (a) nonmonotonic relative vorticity $\zeta\left(\mathrm{s}^{-1}\right)$ and (b) azimuthal velocity $v\left(\mathrm{~m} \mathrm{~s}^{-1}\right)$. (c) Horizontal cross section $(x, y)$ of the thermal pulse $Q\left(\mathrm{~K} \mathrm{~h}^{-1}\right)$ at height $z=5.7 \mathrm{~km}$. (d) Vertical cross section $(x, z)$ of the thermal pulse.

shows snapshots over a selected representative time period $^{3}$ of the normalized $n=2$ dry PV perturbation $q^{\prime}$ (depicted on the horizontal plane). Note that the dry $\mathrm{PV}$ is defined by

$$
q \equiv\left(\zeta_{\theta}+f\right) / \sigma
$$

in which $\zeta_{\theta} \equiv r^{-1} \partial(r v) / \partial r-r^{-1} \partial u / \partial \lambda$ is relative vorticity evaluated at constant $\theta$ and $\sigma \equiv-\partial(p / g) / \partial \theta$ is the isentropic density. From the potential vorticity field, we can see that the asymmetry is made up of one concentric perturbation that is collocated with the negative

\footnotetext{
${ }^{3}$ An ENM analysis verified that the dominant patterns explaining most of the statistical variance in a 12-h simulation period closely resemble the patterns illustrated in Fig. 3 (see the appendix for further details and Fig. 15).
}

maximum of the basic-state radial PV gradient (identified by the dashed blue circle). In time, the asymmetry retains its coherent structure and spins about the vertical axis with nearly a constant phase velocity. This oscillation is interpreted as a discrete VR wave that occurs because of a finite radial gradient of PV. In principle, our nonmonotonic basic-state vortex may accommodate an additional discrete VR wave near the maximum positive PV gradient on the inner edge of the vortex (identified by the dashed yellow circle). Classic barotropic instability relies on the presence of two such waves with the ability to become phase locked so as to induce mutual growth. With this in mind, the presence of only one VR wave suggests that the main destabilizing mechanism in the current case is not barotropic instability.

Moving toward the direction of identifying the dominant instability mechanism, some hints can be obtained 


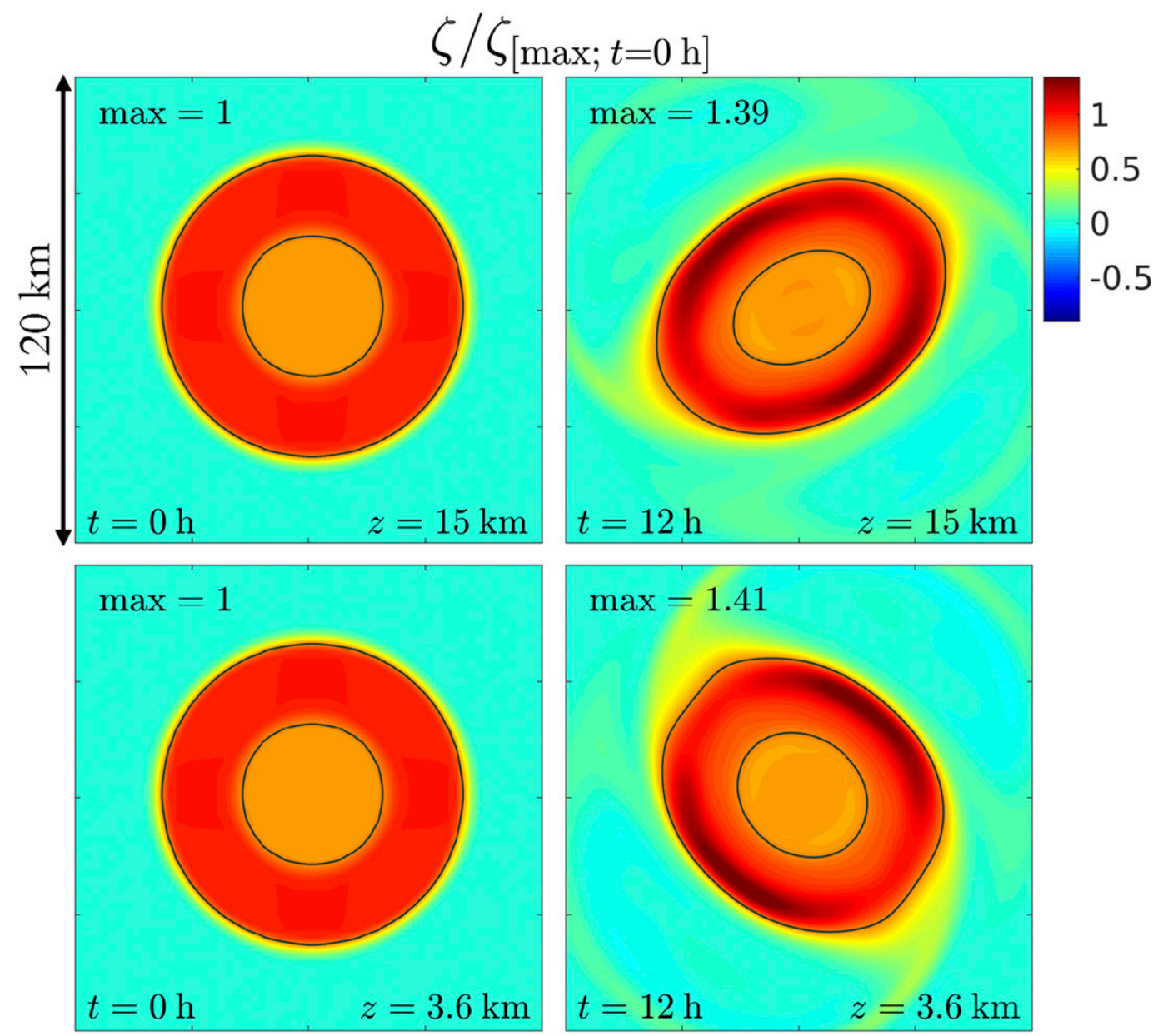

FIG. 2. Horizontal cross sections of normalized relative vorticity $\zeta / \zeta_{[\max ; t=0 \mathrm{~h}]}$ at (left) initial time $t=0 \mathrm{~h}$ and (right) $t=12 \mathrm{~h}$. The cross sections are taken at height (top) $z=15$ and (bottom) $z=3.6 \mathrm{~km}$. The normalization factor $\zeta_{[\max ; t=0 \mathrm{~h}]}$ is the initial maximum value of the relative vorticity.

by looking at the $n=2$ vertical velocity perturbation $w^{\prime}$ (Fig. 3, bottom panels). Here, the asymmetry is composed of two parts. The concentric pattern near the outer edge of the vortex core is the aforementioned VR wave, a footprint of which can be traced in the vertical velocity (note the phase differences between $q^{\prime}$ and $w^{\prime}$; also note the different plotted horizontal scales). The wave pattern in the peripheral of the vortex (where PV gradient is negligible) is suggestive of an outwardpropagating spiral IG wave radiating from the vortex core. The overall structure indicates that the spontaneous imbalance, a mechanism by which a core VR wave amplifies by emitting an outward-propagating IG wave into the environment (e.g., Schecter and Montgomery 2004), might be the main cause of the elliptical deformation. It is worth mentioning that the $e$-folding time of this instability is approximately $3 \mathrm{~h}$, which is sufficiently short to be meteorologically relevant.

\section{Comparisons with a simple linear model}

Following Schecter and Montgomery (2004), the primitive equations linearized about an azimuthal invariant and balanced basic-state vortex, can be written as ${ }^{4}$

$$
\begin{array}{r}
\frac{\partial u^{\prime}}{\partial t}+\bar{\Omega} \frac{\partial u^{\prime}}{\partial \lambda}-\bar{\xi} v^{\prime}+\frac{\partial \phi^{\prime}}{\partial r}=-\nu_{s} u^{\prime} \\
\frac{\partial v^{\prime}}{\partial t}+\bar{\Omega} \frac{\partial v^{\prime}}{\partial \lambda}+\bar{\eta} u^{\prime}+\frac{1}{r} \frac{\partial \phi^{\prime}}{\partial \lambda}=-\nu_{s} v^{\prime}
\end{array}
$$

\footnotetext{
${ }^{4}$ Here, the perturbation equations are formulated in pseudoheight vortex-centered cylindrical coordinates and incorporate hydrostatic and Boussinesq approximations. The variable $\mathrm{z}$ represents pseudoheight as defined in Hoskins and Bretherton (1972) rather than the actual height variable $z$. The basic state of the vortex satisfies gradient wind and hydrostatic balance.
} 

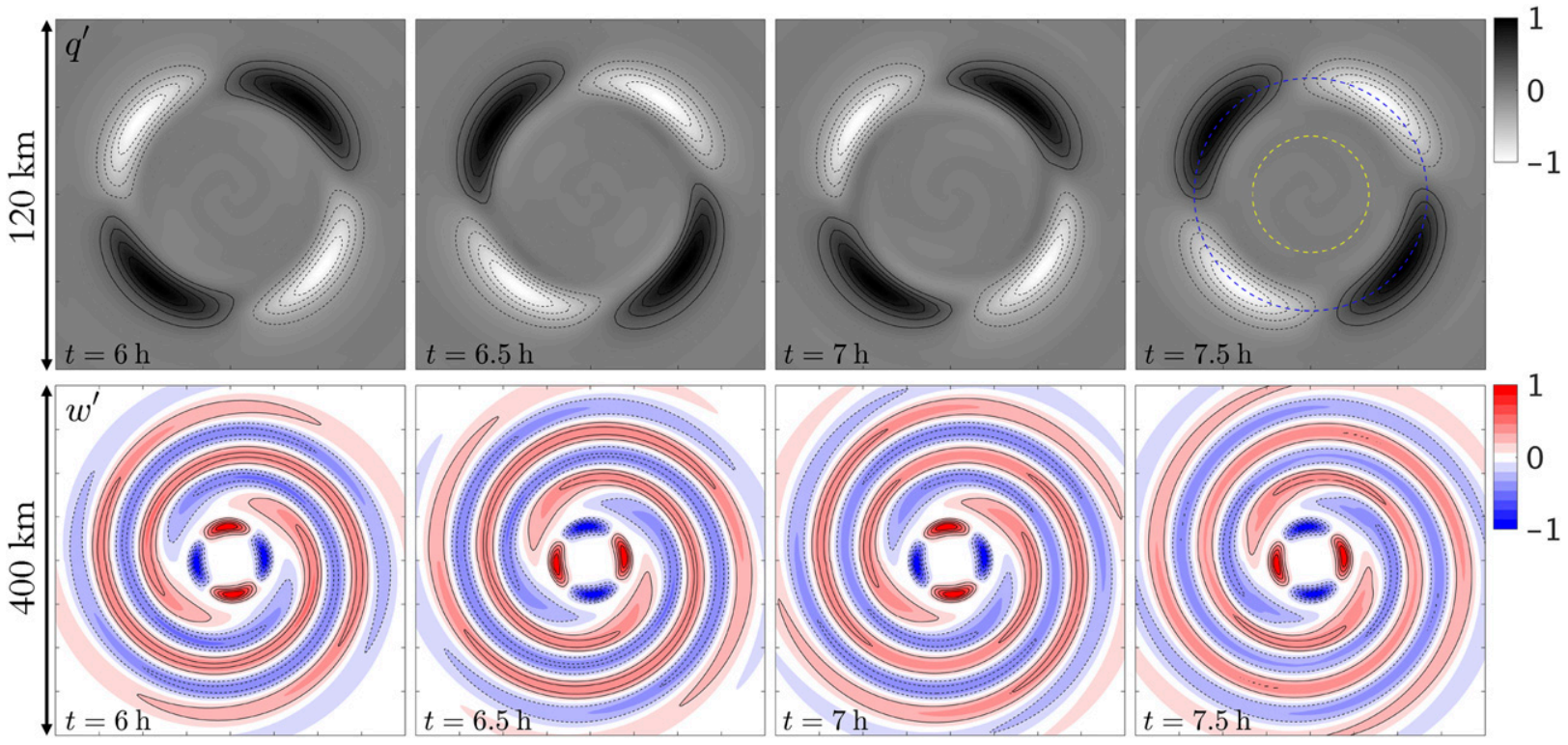

FIG. 3. Snapshots at $t=6,6.5,7,7.5 \mathrm{~h}$ of the (top) azimuthal wavenumber $n=2$ dry potential vorticity perturbation $q^{\prime}$ taken at isentropic-level $\theta=303 \mathrm{~K}$ and (bottom) $n=2$ vertical velocity perturbation $w^{\prime}$ at $\theta=307 \mathrm{~K}$. In any given panel, the plotted values are normalized to the maximum absolute value of the given perturbation field. (top right) The dashed yellow (blue) circle traces the location at which the basic-state radial PV gradient has its maximum positive (negative) value.

$$
\begin{aligned}
& \frac{\partial}{\partial t}\left(\frac{\partial \phi^{\prime}}{\partial \mathrm{z}}\right)+\bar{\Omega} \frac{\partial}{\partial \lambda}\left(\frac{\partial \phi^{\prime}}{\partial \mathrm{z}}\right)+w^{\prime} N^{2}=-\nu_{s}\left(\frac{\partial \phi^{\prime}}{\partial \mathrm{z}}\right), \\
& \frac{1}{r} \frac{\partial\left(r u^{\prime}\right)}{\partial r}+\frac{1}{r} \frac{\partial v^{\prime}}{\partial \lambda}+\frac{\partial w^{\prime}}{\partial \mathrm{z}}=0 .
\end{aligned}
$$

Here, the overbar denotes a basic-state component, and prime denotes a perturbation component. The variables $(u, v, w)$ are the three velocity components in which $u$ is the radial velocity and $\phi$ is the geopotential. The auxiliary field $\Omega \equiv v / r$ is the angular rotation frequency, $\xi \equiv 2 \Omega+f$ is the modified Coriolis parameter, and $\eta \equiv \zeta+f$ is the absolute vorticity. The parameter $N$ is the dry buoyancy frequency, which along with the Coriolis parameter $f$ is treated as a constant. ${ }^{5}$ The parameter $\nu_{s}$ is a circular sponge ring applied at large radii near the outer boundaries.

For isothermal boundaries at $\mathrm{z}=0$ and $H$, the normal modes of the vortex are perturbations of the form

\footnotetext{
${ }^{5}$ The reader is reminded that in contrast to the linear model (which assumes constant $N$ ), the far-field temperature and pressure in the WRF Model is prescribed according to the Jordan (1958) sounding. Better agreement between the two models can be obtained from the following steps: First, the vertical profile of temperature that gives constant $N^{2}$ is obtained analytically. Then the vertical profile of pressure is extracted from the hydrostatic balance. We performed this exercise and subsequently compared the output between two WRF simulations. The results were found to be qualitatively very similar (not shown).
}

$$
\begin{aligned}
u^{\prime}(r, \lambda, \mathrm{z}, t) & =\hat{U}(r, t) \cos (m \pi \mathrm{z} / H) e^{i n \lambda}+\text { c.c. }, \\
v^{\prime}(r, \lambda, \mathrm{z}, t) & =\hat{V}(r, t) \cos (m \pi \mathrm{z} / H) e^{i n \lambda}+\text { c.c. }, \\
\phi^{\prime}(r, \lambda, \mathrm{z}, t) & =\hat{\Phi}(r, t) \cos (m \pi \mathrm{z} / H) e^{i n \lambda}+\text { c.c. }, \\
w^{\prime}(r, \lambda, \mathrm{z}, t) & =\hat{W}(r, t) \sin (m \pi \mathrm{z} / H) e^{i n \lambda}+\text { c.c. },
\end{aligned}
$$

where the circumflex/hat denotes a time-dependent radial wave function and c.c. is the complex conjugate of the term to the left. Substituting the normal-mode solution into Eqs. (4)-(7), the system of equations can be expressed as a linear dynamical system:

$$
\frac{d X}{d t}=\mathbf{T X}
$$

in which the state vector $\mathbf{X} \equiv[\hat{U}, \hat{V}, \hat{\Phi}]^{\mathrm{T}}$ and the time evolution operator $\mathbf{T} \equiv \mathbf{A}^{-1} \mathbf{B}$, where

$$
\begin{aligned}
& \mathbf{A}=\left(\begin{array}{lll}
1 & 0 & 0 \\
0 & 1 & 0 \\
0 & 0 & 1
\end{array}\right), \\
& \mathbf{B}=\left[\begin{array}{ccc}
-\left(i n \bar{\Omega}+\nu_{s}\right) & \bar{\xi} & -\frac{\partial}{\partial r} \\
-\bar{\eta} & -\left(i n \bar{\Omega}+\nu_{s}\right) & -\frac{i n}{r} \\
-Y \frac{\partial(r)}{\partial r} & -i n Y & -\left(i n \bar{\Omega}+\nu_{s}\right)
\end{array}\right],
\end{aligned}
$$


and

$$
Y \equiv \frac{N^{2} H^{2}}{r m^{2} \pi^{2}}
$$

With the above representation, the stability properties of the system can be determined directly from numerical analysis of the matrix $\mathbf{T}^{6}{ }^{6}$ The evolution of an initial asymmetry can be examined by numerically integrating Eq. (9a) forward in time with a fourth-order RungeKutta scheme. In what follows, we will first focus on the dominant eigenmode among the complete set generated by the solver and compare its spatial structure with the patterns extracted from the nonlinear WRF simulation. Then a numerical simulation of the linear equations will be briefly compared with the nonlinear WRF simulation.

\section{a. Linear stability analysis}

For comparison, Fig. 4 shows the $\{n, m\}=\{2,2\}$ most unstable eigenmode of a barotropic vortex with an identical radial structure to the vortex used in the WRF simulation (see Figs. 1a,b), and $f=5 \times 10^{-5} \mathrm{~s}^{-1}$, $N=0.011 \mathrm{~s}^{-1}$, and $H=10 \mathrm{~km}$. The $e$-folding time $\omega_{i}^{-1}$ ( $\omega_{i}$ is the growth rate) of this mode is $2.93 \mathrm{~h}$. The contour plot depicts the vertical velocity perturbation $w^{\prime}$ at an arbitrary value of $\mathrm{z}$ where the vertical wave function is nonzero. Note that $w^{\prime}$ is obtained by computing first the vertical velocity wave function $\hat{W}$ from the following relation:

$$
\hat{W}=-\frac{H}{m \pi}\left(\frac{\partial \hat{U}}{\partial r}+\frac{\hat{U}}{r}+\frac{i n \hat{V}}{r}\right) .
$$

The linear stability analysis reveals that spontaneous IG wave radiation drives the most rapid instability. The spatial structure of this instability closely resembles the structure of the dominant asymmetries extracted from the nonlinear simulation, that is, a discrete azimuthally propagating vortex Rossby wave at the outer edge of the vortex core (there is no discernible presence/participation of an inner-edge vortex Rossby wave) coupled to a spiral IG wave. This favorable comparison provides further confidence that the amplifying elliptical deformation is indeed a result of spontaneous imbalance. While not shown here, it has been verified that the aforementioned $n=2$ unstable radiative eigenmode

\footnotetext{
${ }^{6}$ The system is discretized on a staggered radial grid that is made up of two subgrids. Details about the staggering can be found in appendix A of Menelaou et al. (2016). The domain extends from $r=0$ to $r=220 \mathrm{~km}$ on a regularly spaced grid with $100-\mathrm{m}$ grid spacing.
}

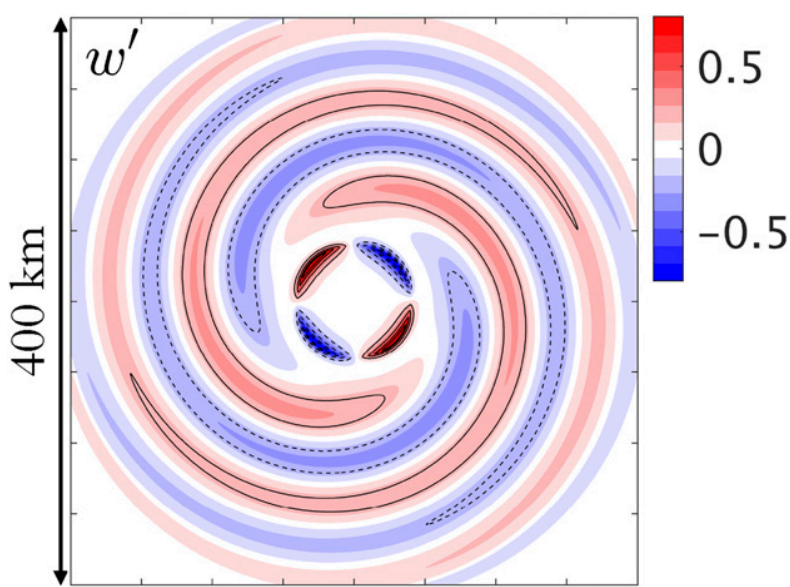

FIG. 4. The vertical velocity perturbation $w^{\prime}$ (at arbitrary z) associated with the $n=2$ dominant eigenmode. The color scale is normalized to the maximum plotted value of $w^{\prime}$.

dominates also in a generic asymmetric instability in which the initial perturbation has no preference toward a particular value of $n$ (between 1 and 8). Of interest to note is that the dominant unstable modes of both the second and third most important $n$ (which is found respectively to be 3 and 4 , with 3 possessing high growth rates close to those of $n=2$; their $e$-folding times are, respectively, 2.96 and $3.35 \mathrm{~h}$ ) are also radiative. With this in mind, it is reasonable to hypothesize that if the initial thermal perturbation is biased toward one of these wavenumbers, spontaneous imbalance may deform the vortex core into either a triangular or a square shape (more discussion on this matter will be provided later).

\section{b. Linear numerical simulation}

With supporting evidence from the above stability analysis, the nonlinear WRF simulation is envisioned to evolve as follows: The initial small thermal perturbation excites one discrete baroclinic VR wave by deforming the mean PV distribution. In time, the VR wave emits a frequency-matched spiral IG wave into the environment. The emission has positive feedback on the VR wave, causing both to grow.

Perhaps nothing says more about the actual dominance of this linear instability mechanism in the WRF simulation as the way it compares to a linear simulation. Figure 5 (top panels) shows the response of the linear model [Eq. (9a)] to a slight elliptical deformation introduced in the geopotential field. The structure of the deformation resembles the one used to perturb the WRF Model. For comparison, the response of the WRF Model at the same time period is also shown (bottom panels). We focus on the similarities and differences between the dominant spatial patterns as the two models 


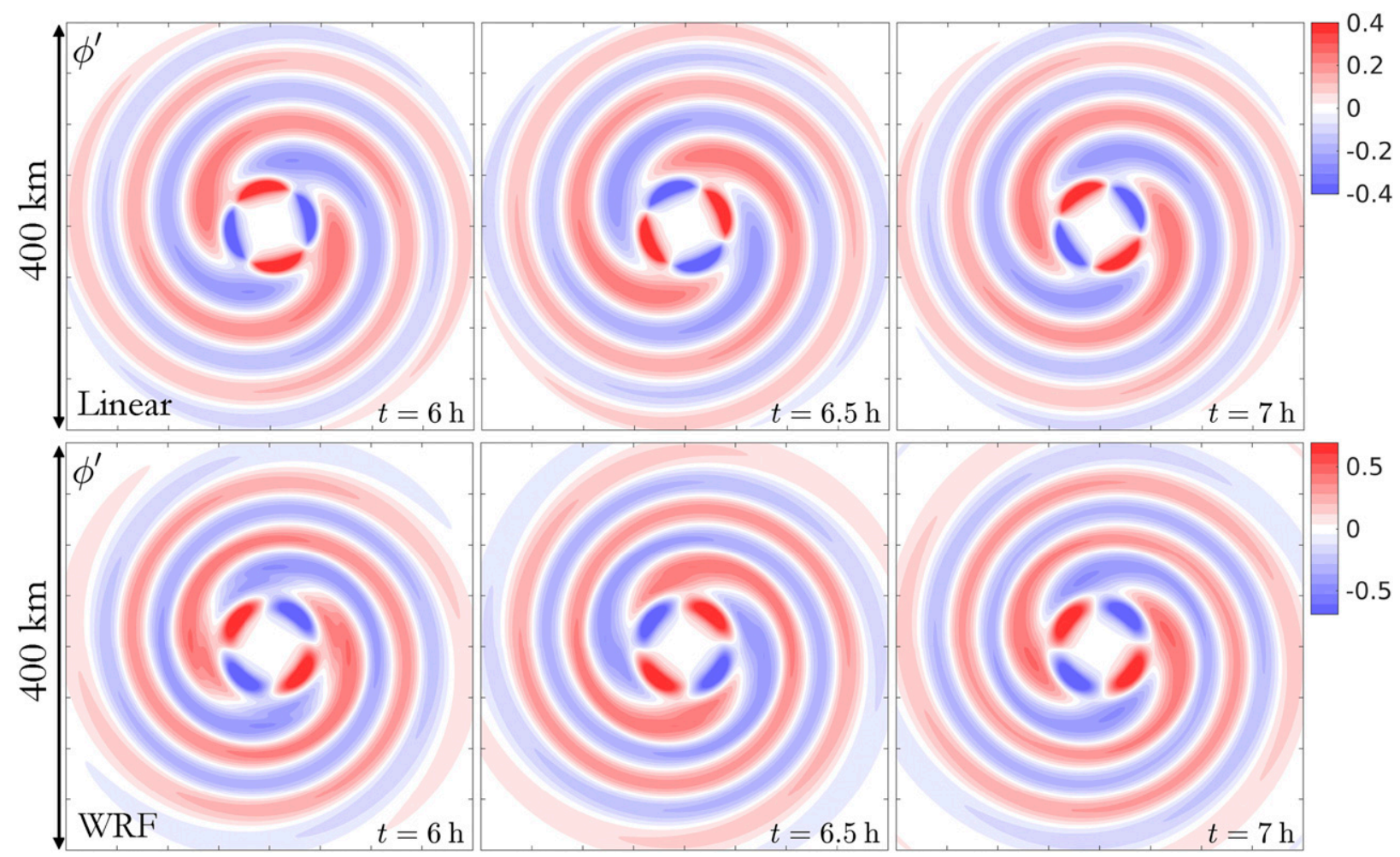

FIG. 5. Snapshots at $t=6,6.5,7 \mathrm{~h}$ of the $n=2$ geopotential perturbation $\phi^{\prime}$. (top) Linear model response (at arbitrary z). (bottom) WRF Model response (at $\theta=307 \mathrm{~K}$ ). In any given panel, the plotted values are normalized to the given maximum absolute value of $\phi^{\prime}$.

exhibit distinct differences that prevent in-depth quantitative comparison. With that being said, after a brief transition period, the most unstable $(n=2)$ eigenmode dominates the perturbation in the linear integration. The geopotential perturbation $\phi^{\prime}$ field reveals striking similarities between the two models, both in terms of their spatial patterns and angular phase velocity. This resemblance verifies with little doubt that the dominant destabilizing mechanism responsible for the elliptical deformation in the nonlinear WRF Model is the spontaneous radiation of spiral IG waves by a core discrete VR wave.

\section{Wave activity diagnostics}

To physically understand how the core VR wave in the WRF simulation amplifies as a result of producing radiation, we turn to wave activities. The prominent equation we consider in the discussion is the lowest-order flux conservative equation for azimuthal-integrated angular pseudomomentum density $\mathcal{J}$ associated with the $n=2$ disturbance. In isentropic coordinates,

$$
\frac{\partial \mathcal{J}}{\partial t}=\nabla \cdot \mathbf{F},
$$

in which,

$$
\mathcal{J} \equiv \mathcal{J}^{q}+\mathcal{J}^{v \sigma},
$$

where

$$
\begin{aligned}
\mathcal{J}^{q} & \equiv-\int_{0}^{2 \pi} d \lambda \frac{r \bar{\sigma}^{2}\left(q^{\prime}\right)^{2}}{2 \partial \bar{q} / \partial r}=-\frac{2 \pi r \bar{\sigma}^{2}\left|q_{2}\right|^{2}}{\partial \bar{q} / \partial r}, \\
\mathcal{J}^{v \sigma} & \equiv-\int_{0}^{2 \pi} d \lambda r v^{\prime} \sigma^{\prime}=-4 \pi r \mathcal{R}\left[\sigma_{2} v_{2}^{*}\right]
\end{aligned}
$$

and

$$
\nabla \cdot \mathbf{F} \equiv \frac{1}{r} \frac{\partial}{\partial r}\left(r F_{r}\right)+\frac{\partial}{\partial \theta}\left(F_{\theta}\right)
$$

where

$$
\begin{aligned}
& F_{r} \equiv \int_{0}^{2 \pi} d \lambda r \bar{\sigma} u^{\prime} v^{\prime}=4 \pi r \bar{\sigma} \mathcal{R}\left[u_{2} v_{2}^{*}\right] \\
& F_{\theta} \equiv-\int_{0}^{2 \pi} d \lambda \frac{p^{\prime}}{g} \frac{\partial M^{\prime}}{\partial \lambda}=\frac{4 \pi n}{g}\left(\mathcal{R}\left[p_{2}\right] \mathcal{I}\left[M_{2}\right]-\mathcal{I}\left[p_{2}\right] \mathcal{R}\left[M_{2}\right]\right)
\end{aligned}
$$



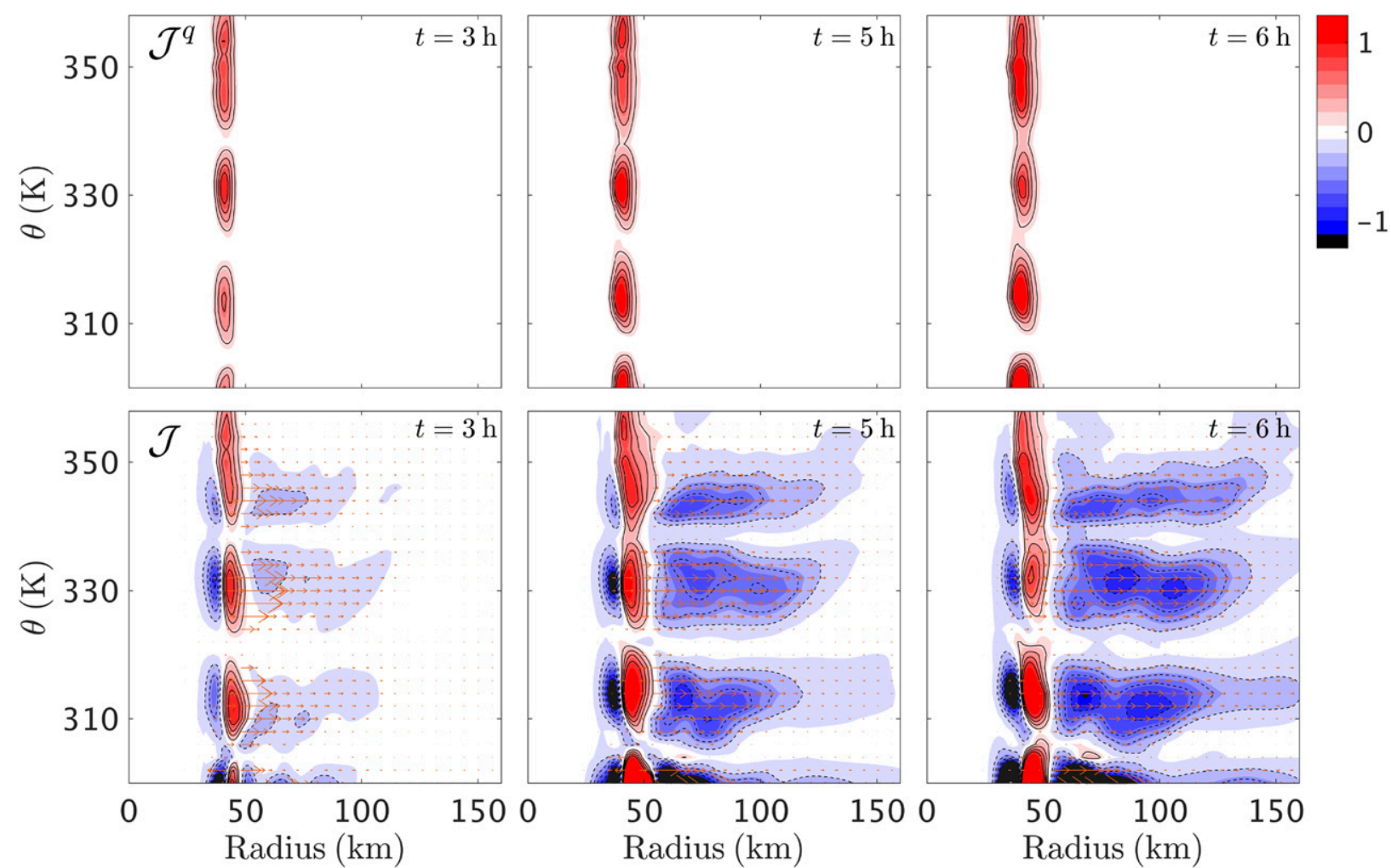

FIG. 6. Snapshots at $t=3,5,6 \mathrm{~h}$ of the (top) PV component of angular pseudomomentum density $\mathcal{J}^{q}$ and (bottom) total angular pseudomomentum density $\mathcal{J}$. The arrows superposed on the contour plots of $\mathcal{J}$ is the wave activity flux vector $\mathbf{F}$. The values in all panels are scaled to the maximum value of $\mathcal{J}^{q}$ at $t=3 \mathrm{~h}$.

Here, $M \equiv c_{p} T+\phi$ is the Montgomery streamfunction, in which $c_{p}$ is the specific heat of dry air at constant pressure and $T$ is temperature. The subscript 2 indicates the wavenumber- 2 component, the asterisk superscript indicates the complex conjugate, and $\mathcal{R}[\cdots]$ and $\mathcal{I}[\cdots]$ denotes the real and imaginary parts of the quantity in square brackets, respectively.

The $\mathcal{J}^{q}$ component contains the square PV perturbation and radial $\mathrm{PV}$ gradient and tends to dominate the angular pseudomomentum of a VR wave (Chen et al. 2003). On the other hand, the $\mathcal{J}^{v \sigma}$ component is proportional to $v^{\prime} \sigma^{\prime}$ and tends to dominate the angular pseudomomentum of an IG wave. The parameter $F_{r}$ is proportional to the perturbation radial angular momentum flux, and the parameter $F_{\theta}$ is proportional to the perturbation radial heat flux. ${ }^{7}$ If the pseudomomentum flux vector $\mathbf{F}$ points radially outward, perturbation angular momentum is transported outward, whereas if $\mathbf{F}$ points vertically upward, then perturbation heat is transported outward, and vice versa. Divergence of the flux vector signifies the local rate of change of wave

\footnotetext{
${ }^{7}$ In pressure coordinates, $F_{\theta}$ is analogous to $\int_{0}^{2 \pi} d \lambda u^{\prime} T^{\prime}$.
}

activity. When $\nabla \cdot \mathbf{F}>0$, angular pseudomomentum locally increases, whereas when $\nabla \cdot \mathbf{F}<0$, then angular pseudomomentum locally decreases.

Figure 6 portrays the basic structure of angular pseudomomentum at different times. The top panels are contour plots of the PV component $\mathcal{J}^{q}$. The bottom panels show superposed contour plots of the total pseudomomentum $\mathcal{J}$ and wave activity flux vector $\mathbf{F}$. Looking at $\mathcal{J}^{q}$, only the core VR wave can be identified. Its wave activity is positive since the local gradient $\partial \bar{q} / \partial r<0$. If the VR wave were situated near the inner edge of the vortex core, it is not difficult to imagine that $\mathcal{J}^{q}$ would have been negative (since local $\partial \bar{q} / \partial r>0$ ). Of interest to note is the coherent baroclinic nature of this wave, which accounts for the vertical structure of the elliptical eyewall.

Looking at $\mathcal{J}$, the picture is quite different. The IG wave is now clearly visible in the periphery of the vortex core (outside a radius of about $50 \mathrm{~km}$ ), almost attached to the VR wave. This feature along with a baroclinic structure nearly in phase with the VR wave is suggestive of an IG wave that is indeed emitted by the core VR wave. In contrast to the VR wave, the negative $\mathcal{J}^{v \sigma}$ component dominates the IG wave activity. The wave 


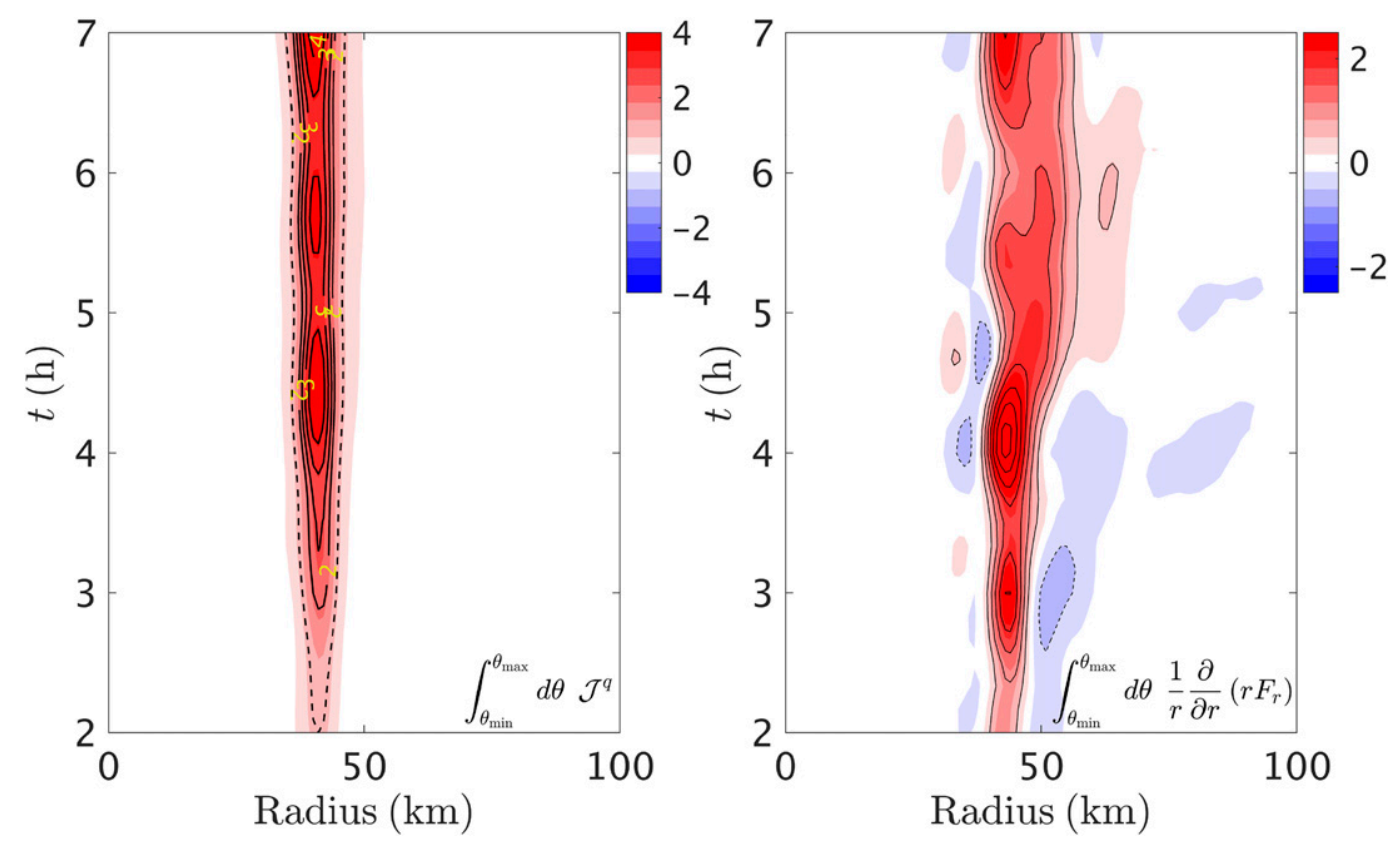

FIG. 7. (left) Radius-time diagram of the vertically integrated $\mathcal{J}^{q}$. The values are scaled to the maximum plotted value at $t=2 \mathrm{~h}$. The dashed contour traces values equal to 1 . Solid contours range from 2 to 4 with a 0.5 interval. (right) Radius-time diagram of the vertically integrated divergence of radial wave activity fluxes. The values are scaled to the maximum plotted value at $t=2 \mathrm{~h}$.

activity flux vector (which originates near the transition zone between the VR and IG wave) is collocated with the IG wave and points radially outward. This suggests that IG wave radiation involves the ejection of negative wave activity from the cyclone into the environment (consistent with previous studies; e.g., Schecter and Montgomery 2004).

As mentioned previously, the divergence of the wave activity fluxes determines the local rate of change of wave activity. Figure 7 shows the time evolution of the vertically integrated $\mathcal{J}^{q}$ (left panel) and the divergence of the radial wave activity fluxes (right panel). It can be seen that the VR wave amplifies with time. The flux divergence induced by the IG wave radiation largely accounts for this amplification. In other words, the core VR wave and thus the elliptical deformation of the eyewall spontaneously amplifies as a result of producing radiation.

At this point, it is worth remarking that one may additionally view the growth of the core VR wave to be a consequence of conservation of wave activity (cf. Schecter and Montgomery 2004). Recall that wave activities define global invariants when the net flux across the boundaries vanish. For discussion purposes, let us assume a cylindrical volume large enough for this assumption to hold. The total angular pseudomomentum $\mathcal{W}$ is the integral

$$
\mathcal{W} \equiv \int_{\theta_{b}}^{\theta_{t}} \int_{0}^{r_{v}} d \theta d r r J
$$

where $r_{v}$ is the radius of the cylinder and $\theta_{b}$ and $\theta_{t}$ denote the isentropes where the bottom and top surfaces of the cylinder are situated, respectively. The aforementioned analyses further suggest that it is reasonable to decompose the total angular pseudomomentum into two regional components: the vortex core associated with the VR wave (core) and its environment associated with the spiral IG wave (env); that is, $\mathcal{W}=\mathcal{W}_{\text {core }}+\mathcal{W}_{\text {env }}$, in which

$$
\begin{gathered}
\mathcal{W}_{\text {core }} \equiv \int_{\theta_{b}}^{\theta_{t}} \int_{0}^{r_{c}} d \theta d r r J, \\
\mathcal{W}_{\text {env }} \equiv \int_{\theta_{b}}^{\theta_{t}} \int_{r_{c}}^{r_{v}} d \theta d r r J .
\end{gathered}
$$

Here, $r_{c}$ defines the vortex-core radius. Integrating Eq. (11) over this volume, we obtain the conservation law:

$$
\frac{d \mathcal{W}_{\text {core }}}{d t}=-\frac{d \mathcal{W}_{\text {env }}}{d t}
$$

The wave activity within the vortex environment was shown to be systematically negative and to amplify with time (see Fig. 6). Equation (18) states that the core wave activity must grow to compensate for these losses. 


\section{Higher azimuthal wavenumber thermal pulse}

As briefly mentioned, a linear stability analysis for generic asymmetric instabilities revealed that the elliptical radiative eigenmode is the most dominant. This implies, for instance, that if the current basic-state vortex within a WRF simulation is perturbed by an initial thermal pulse with no preference toward a particular wavenumber, then $n=2$ spontaneous radiative imbalance should be expected to dominate, deforming its core into an ellipse. In fact, this was verified with two independent WRF simulations in which the initial pulse was constructed from the superposition of azimuthal wavenumbers 1-8 of equal amplitude (and with an overall maximum amplitude of $5 \mathrm{~K} \mathrm{~h}^{-1}$ in the first simulation and $1 \mathrm{~K} \mathrm{~h}^{-1}$ in the second). For example, Fig. 8 shows the time evolution (from the second WRF simulation) of the maximum amplitude of select components from the Fourier expansion $(n=1,8)$ of vertical velocity. Here, it can be seen that early in the simulation, the dominant wavenumbers appear to be 2 and 3. However, as time evolves, the $n=2$ perturbation clearly dominates the overall asymmetry. Perhaps one notable difference worth mentioning is that in these simulations, the onset of spontaneous imbalance appears to be delayed for a few hours relative to when the pulse was prescribed with an $n=2$ spatial structure.

On the other hand, linear stability analysis also revealed that other azimuthal wavenumber perturbations might possess significant (although secondary) growth rates. In particular, the second most unstable wavenumber for our control cyclone is characterized by $n=3$, followed by $n=4$. Of interest, for both wavenumbers, the dominant modes are radiative. This suggests that if the initial perturbation is biased toward one of these $n$, then spontaneous imbalance might deform the vortex core into other shapes besides ellipses. To test whether this actually occurs, two additional simulations are presented: one in which the pulse has an $n=3$ structure and one with $n=4$ structure. The maximum amplitude, vertical structure, and activation time remains the same as in the control case.

Figure 9 depicts the vortex structure after $5.5 \mathrm{~h}$ of simulation in terms of total PV $q$ and the resultant dominant asymmetries (in terms of PV $q^{\prime}$ and vertical velocity $\left.w^{\prime}\right)$. The left column corresponds to the $n=3$ experiment and the right column to the $n=4$. Looking at $q$, it is seen that the vortex core has lost its initial symmetry and deformed into either a triangular or a square shape. In agreement with findings from the control experiment, this amplifying (not shown) deformation is the result of spontaneous radiative pumping of one baroclinic VR wave (with either $n=3$ or 4 ; see $q^{\prime}$ and $\left.w^{\prime}\right)$. In other words, a key point here is that

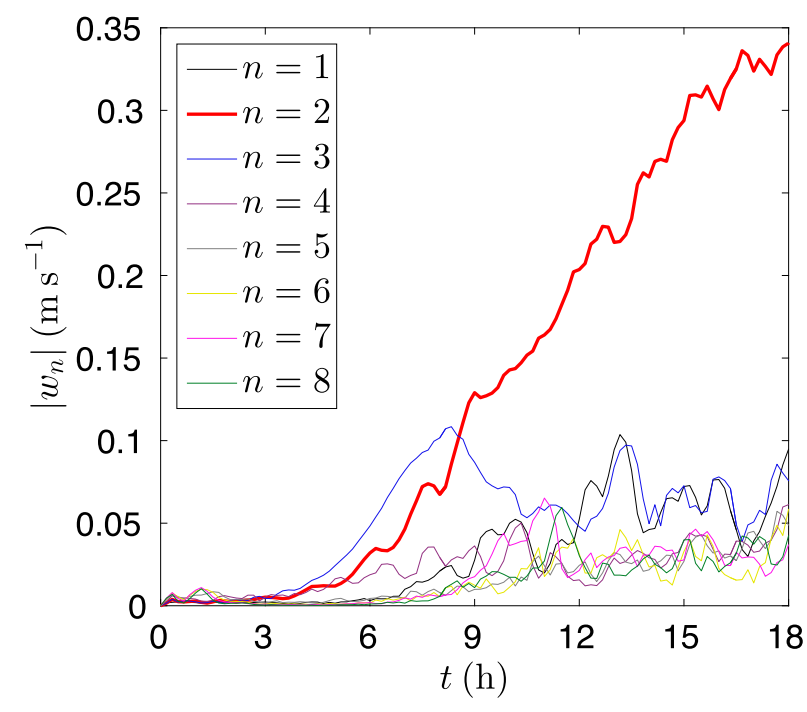

FIG. 8. Time series of the maximum amplitude (within the vortex core $r<55 \mathrm{~km}$ ) of select components from the Fourier expansion of vertical velocity.

spontaneous imbalance is not only restricted to elliptical deformations, but it can remain the dominant destabilizing mechanism behind higher-azimuthal-wavenumber eyewall deformations. ${ }^{8}$

\section{Long-term effect of nonlinear spontaneous imbalance}

To complete this part of the discussion, we briefly examine the long-term effect of nonlinear spontaneous imbalance. It should be recalled that a common longterm effect of traditional nonlinear barotropic instability involves intricate vortex merger and mixing processes that relax the initial annular vortex into one with a monotonic distribution (e.g., Schubert et al. 1999). It is intriguing to investigate whether a similar outcome can be induced also by an instability with a radiative character.

Figure 10 illustrates the long-term evolution of the control cyclone perturbed by an $n=2$ thermal pulse in terms of total relative vorticity $\zeta$ at a representative vertical level. For reference, the initial vortex structure $(t=0 \mathrm{~h})$ is also depicted. It can be seen that the

\footnotetext{
${ }^{8}$ It is worth mentioning that for the particular control cyclone, perturbations with azimuthal wavenumbers $5 \leq n \leq 8$ exhibit relatively smaller growth rates with respect to large-scale instabilities with $n=2$ or 3 . Independent WRF simulations perturbed by thermal pulses prescribed to have one of these wavenumbers (between 5 and 8 ) resulted in the excitation of a radiative elliptical mode, and no sign of high- $n$ core deformations were observed (not shown).
} 

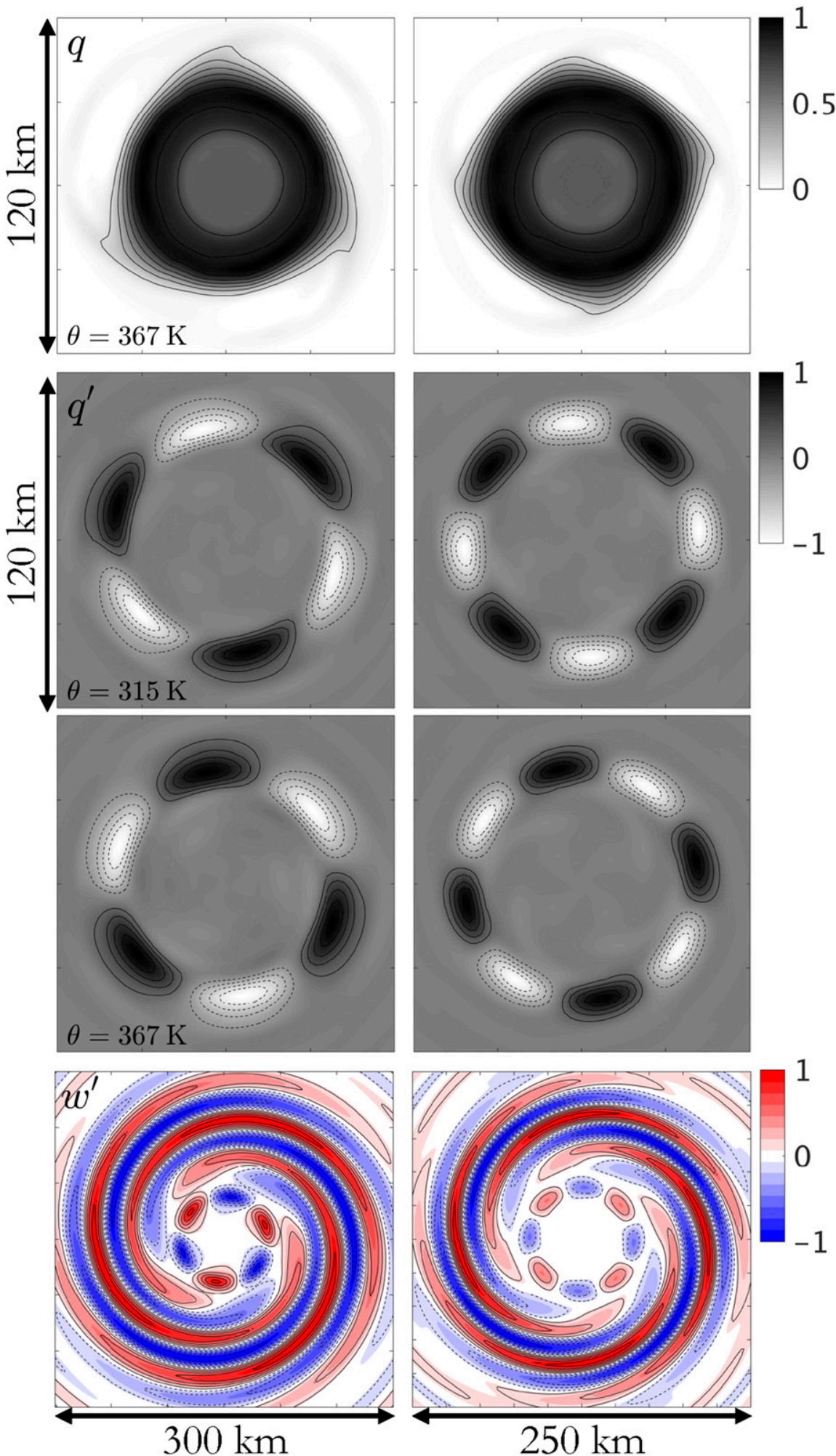

FIG. 9. Snapshots at $t=5.5 \mathrm{~h}$ of (first row) $q$ at $\theta=367 \mathrm{~K}$, (second row),(third row) $q^{\prime}$ at $\theta=315$, $367 \mathrm{~K}$, and (fourth row) $w^{\prime}$ at $\theta=307 \mathrm{~K}$. (left) Experiment with an $n=3$ thermal pulse. (right) Experiment with an $n=4$ thermal pulse. In any given panel, the plotted values are normalized to the maximum value of the given field. 

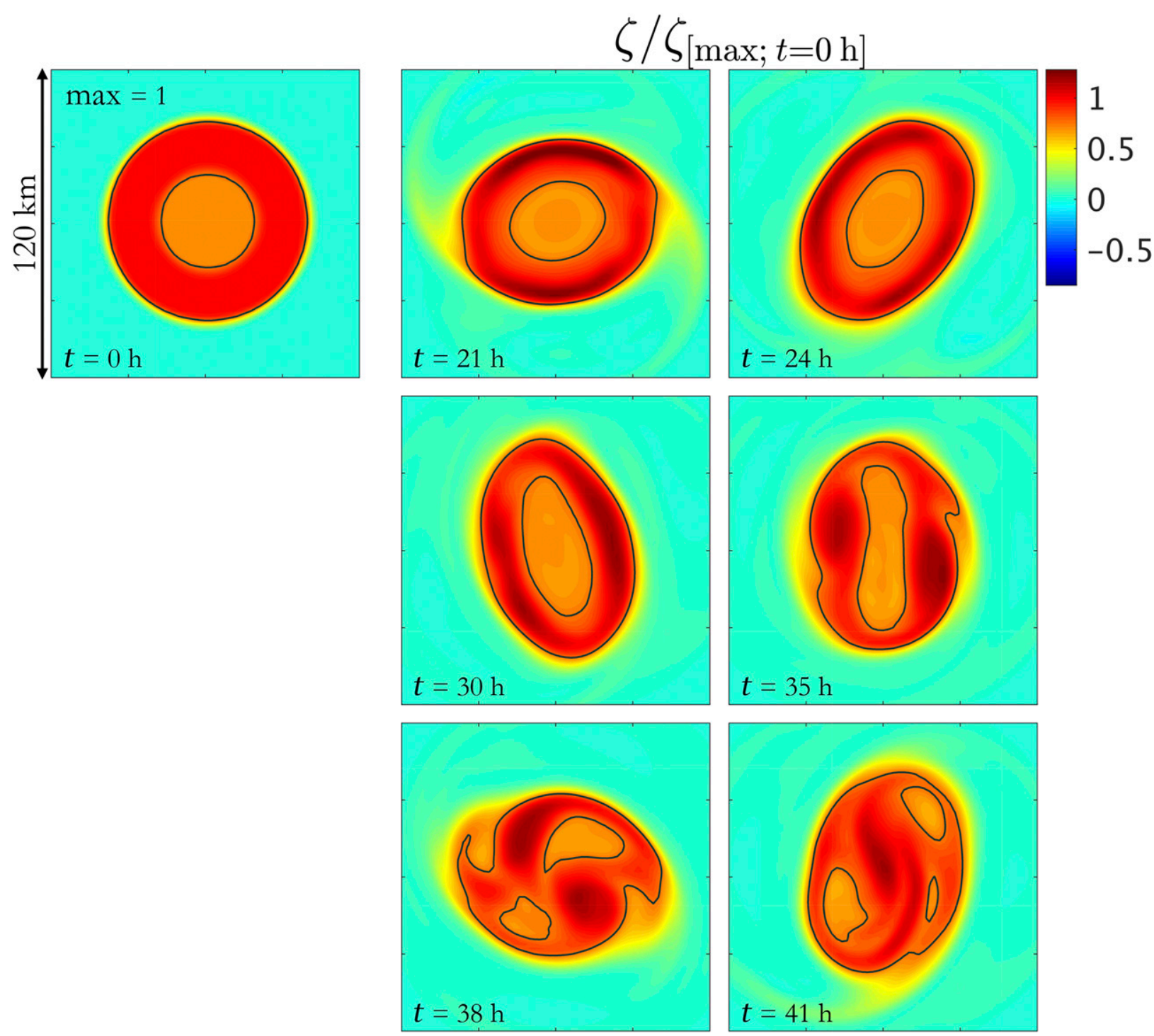

FIG. 10. Long-term nonlinear evolution of the control cyclone perturbed by an initial $n=2$ thermal pulse. (top left) Horizontal cross section of normalized relative vorticity $\zeta / \zeta_{[\max ; t=0 \mathrm{~h}]}$ at initial time $t=0 \mathrm{~h}$. (center column),(right column) Normalized relative vorticity vs time. The cross sections are taken at height $z=8.85 \mathrm{~km}$. The normalization factor $\zeta_{[\max ; t=0 \mathrm{~h}]}$ is the initial maximum value of the relative vorticity.

dominant elliptical mode is preserved for more than $24 \mathrm{~h}$ until the formation of two distinct mesovortices $(t=$ $35 \mathrm{~h})$. Following this, the two mesovortices undergo a merging process leading to a nearly monopolar vorticity distribution by $t=41 \mathrm{~h}$. The evolution of the control cyclone perturbed by the second most significant wavenumber $(n=3)$ evolves in a similar manner. Figure 11 illustrates its long-term nonlinear evolution. Like the previous case, the triangular mode remains the dominant until the formation of three distinct mesovortices. The mesovortices again undergo merging, thereby relaxing the vortex into a monopole $(t=42 \mathrm{~h})$.
Finally, it should be noted that the appearance of mesovortices along with their subsequent merging (although incomplete by the end of a 48-h simulation) is also observed even when the cyclone is perturbed by an equivalent $n=4$ thermal pulse (not shown). In this case, however, the longterm evolution appears to be more complicated because of the influence of modes with different wavenumbers. Here, although the $n=4$ mode remains the dominant for more than $9 \mathrm{~h}$ in the simulation, it transitions into an equivalent $n=3$ mode by $18 \mathrm{~h}$. Eventually, the vortex splits into a number of vortices, which seems to be an outcome of the interaction between $n=3$ and 4 modes together. 

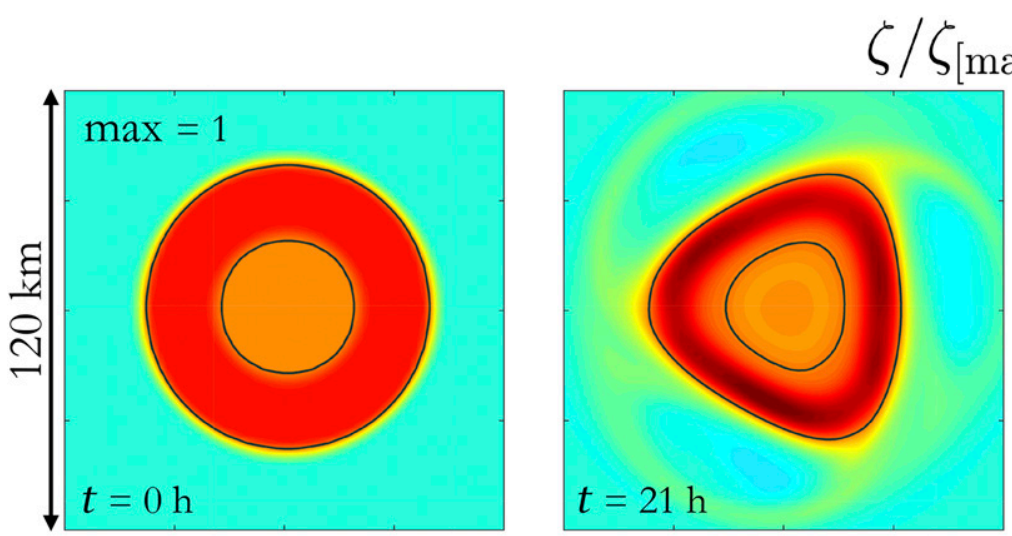

$\max ;=0 \mathrm{~h}]$
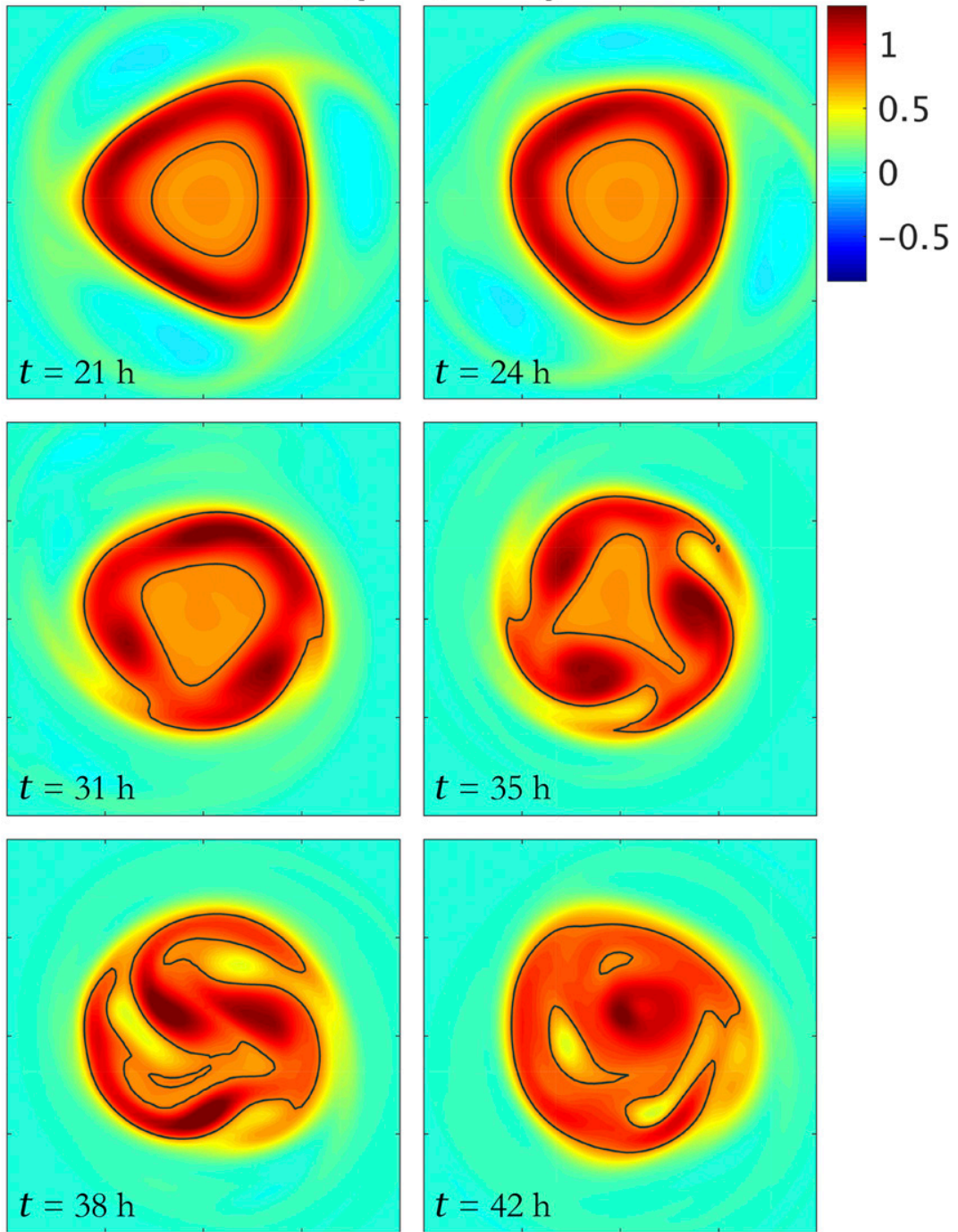

FIG. 11. As in Fig. 10, but for a simulation in which the control cyclone is perturbed by an initial $n=3$ thermal pulse.

\section{Sensitivity experiments}

To this point, we have considered a cyclone with a particular annular vorticity distribution. We remind that annular vortices support the possibility of more than one instability, with traditional barotropic instability considered the most frequently excited. Despite this, we provided evidence of spontaneous IG wave radiation being the fastest destabilizing mechanism (within the nonlinear WRF simulation), dominating the growth of an elliptical deformation for certain vorticity profiles. Here, the extent to which spontaneous imbalance surpasses barotropic instability is further examined with a number of sensitivity tests performed on two pertinent vortex shape parameters: the annulus thickness controlled by the parameter $\mu$ in Eq. (1) and the depth of the central vorticity hole (occasionally referred to as the hollowness) controlled by the parameter $\beta$. The growth rates for coupled VR wave barotropic instability tend to increase as the central vorticity hole becomes deeper (steeper radial PV gradient) and as the vortex annulus becomes thinner (e.g., Schubert et al. 1999). Physically, thinner annuli imply a shorter separation distance between the two coupled VR waves and thus relatively stronger radial PV fluxes across each wave by the other (and therefore higher growth rates).

Figure 12 depicts the initial vortex structure (in terms of relative vorticity) specified in each sensitivity case. The experiments are grouped into three categories. In the first category (Fig. 12a), the vortex annulus is gradually made thinner while leaving its hollowness unaffected (similar to 

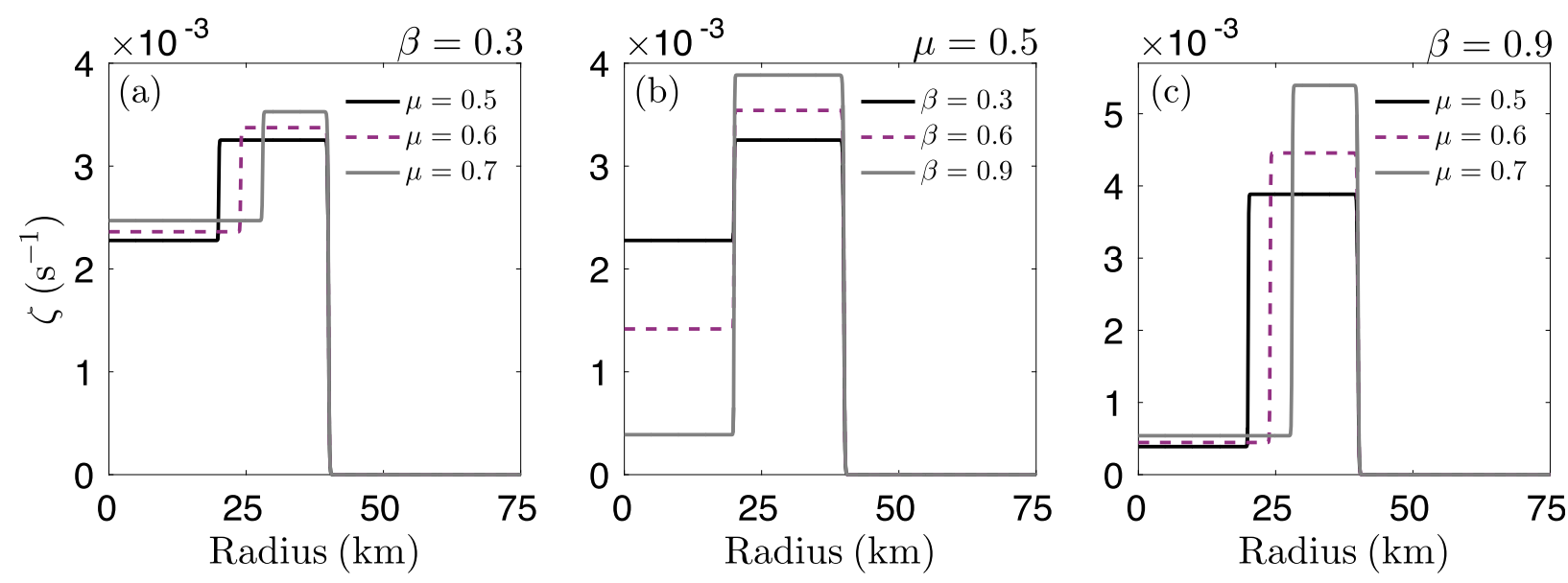

FIG. 12. Variability of the relative vorticity distribution $\zeta(r ; \beta, \mu)$ of the nonmonotonic vortex defined by Eq. (1).

the control experiment, which is illustrated by the black curve). In the second category (Fig. 12b), only the vortex hollowness is modified by gradually becoming deeper. The third category (Fig. 12c) considers a deep hollow vortex with an annulus that is gradually made thinner. Note that the magnitude of vorticity in each experiment is scaled so as to give the same maximum azimuthal velocity $\left(60 \mathrm{~m} \mathrm{~s}^{-1}\right)$. Also note that all vortices are perturbed using the same asymmetric $n=2$ thermal pulse as in the control experiment.

Figure 13 displays snapshots of the resultant asymmetry for the first category in terms of the $n=2 \mathrm{PV}$ perturbation (depicted at three different isentropic levels) and the $n=2$ vertical velocity perturbation. All snapshots are taken at $6.5 \mathrm{~h}$ within a simulation, and the illustrated patterns are representative of the spatial structure of the dominant asymmetry. Each column corresponds to a different case characterized by an initial vortex with a different annulus thickness. Turning to the PV perturbation, it is immediately seen that the resultant patterns exhibit profound similarities. In all cases, the asymmetry resembles only one baroclinic outer-edge VR wave, thereby excluding the possibility of barotropic instability being in action. Comparing the pattern orientation between different isentropic levels, it is also seen that the asymmetries are either nearly in phase or roughly $180^{\circ}$ out of phase. Such a phase relation further rules out the presence of baroclinic instability. As to the vertical velocity perturbation, the patterns are again very similar for the three cases. Here, the asymmetry clearly resembles the outer-edge VR wave coupled to a spiral IG wave indicating that spontaneous imbalance remains the dominant destabilizing mechanism for this group of experiments.

Switching to the second category, the structure of the dominant asymmetry is portrayed in Fig. 14. Each column now corresponds to a vortex with different hollowness, ranging from shallow (left column: control case $\beta=0.3$ ) to intermediate (middle column: $\beta=0.6$ ) to deep (right column: $\beta=0.9$ ). If we focus at first on the shallow and intermediate cases, it seems that doubling the initial hollowness does not significantly alter the results (relative to the control case). Spontaneous radiation of a spiral IG wave from the outer-edge VR wave remains the fastest-growing instability. On the other hand, the deep hollow case results are somewhat different. Here, the perturbation PV pattern inside the vortex resembles that of two VR waves involved in some degree of mutual amplification. The signal of the inneredge VR wave becomes more pronounced at higher levels where the two wave patterns appear to be nearly $90^{\circ}$ out of phase. This is primarily an outcome of the initial vortex structure, which regardless of being barotropic has more of a top-heavy basic-state PV distribution as a result of the vertical variation of basic-state density. Despite this, the vertical velocity perturbation is still suggestive of spontaneous IG wave emission from the outer-edge VR wave. Of interest is that the overall structure resembles an unstable mode previously documented in Menelaou et al. (2016), which indicates that the instability may be viewed as radiative pumping of an outer VR wave boosted primarily by its simultaneous interaction with an inner VR wave.

From the above experiment, it appears that deep hollow vortices with the particular maximum velocity and ambient conditions fall within a regime where both instabilities of interest (barotropic and radiative) may be simultaneously in action. The third category of sensitivity tests is tailored to provide some insight on how differently a vortex might evolve under this regime if additionally one modifies the annuli thickness. 

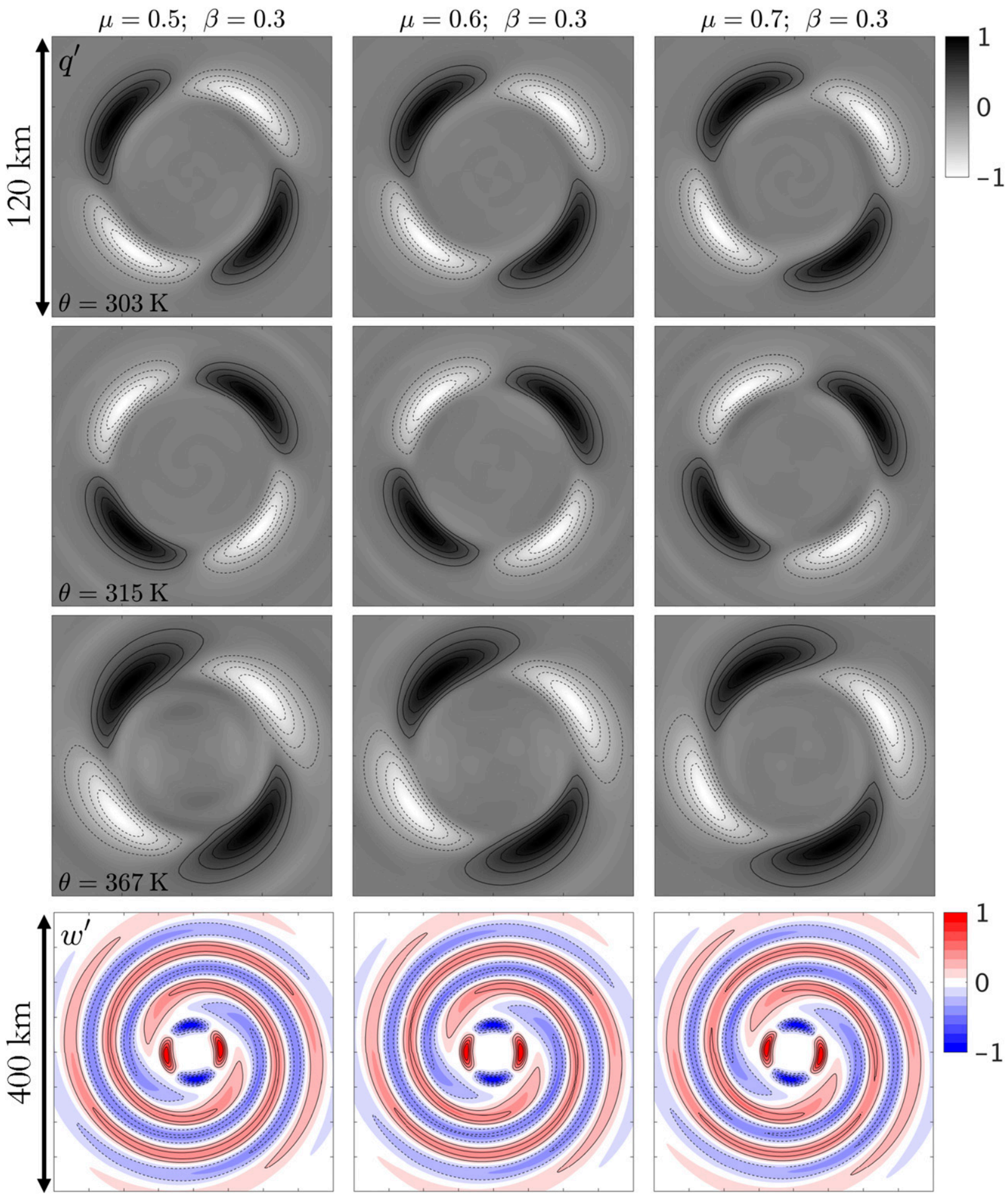

FIG. 13. First category of sensitivity experiments. Snapshots at $t=6.5 \mathrm{~h}$ of the $n=2$ (first row),(second row),(third row) $q^{\prime}$ at $\theta=303$, $315,367 \mathrm{~K}$, and (fourth row) $w^{\prime}$ at $\theta=307 \mathrm{~K}$. The vortex parameters are (left) $\mu=0.5, \beta=0.3$; (middle) $\mu=0.6, \beta=0.3$; and (right) $\mu=0.7, \beta=0.3$. In any given panel, the plotted values are normalized to the maximum absolute value of the given perturbation field. 

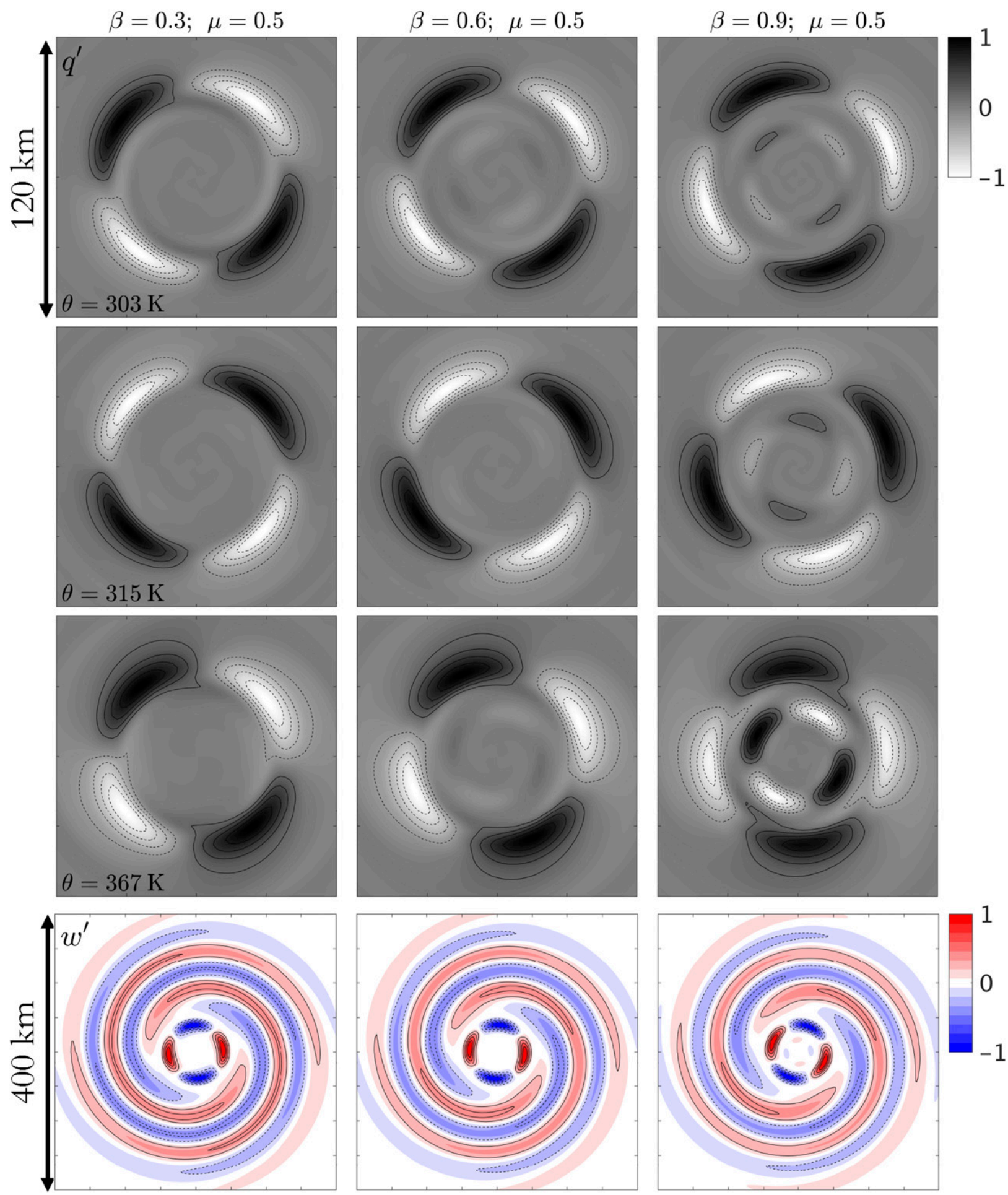

FIG. 14. Second category of sensitivity experiments. Snapshots at $t=5.5 \mathrm{~h}$ of the $n=2$ (first row),(second row),(third row) $q^{\prime}$ at $\theta=303$, $315,367 \mathrm{~K}$, and (fourth row) $w^{\prime}$ at $\theta=307 \mathrm{~K}$. The vortex parameters are (left) $\beta=0.3, \mu=0.5$; (middle) $\beta=0.6, \mu=0.5$; and (right) $\beta=0.9, \mu=0.5$. In any given panel, the plotted values are normalized to the maximum absolute value of the given perturbation field. 

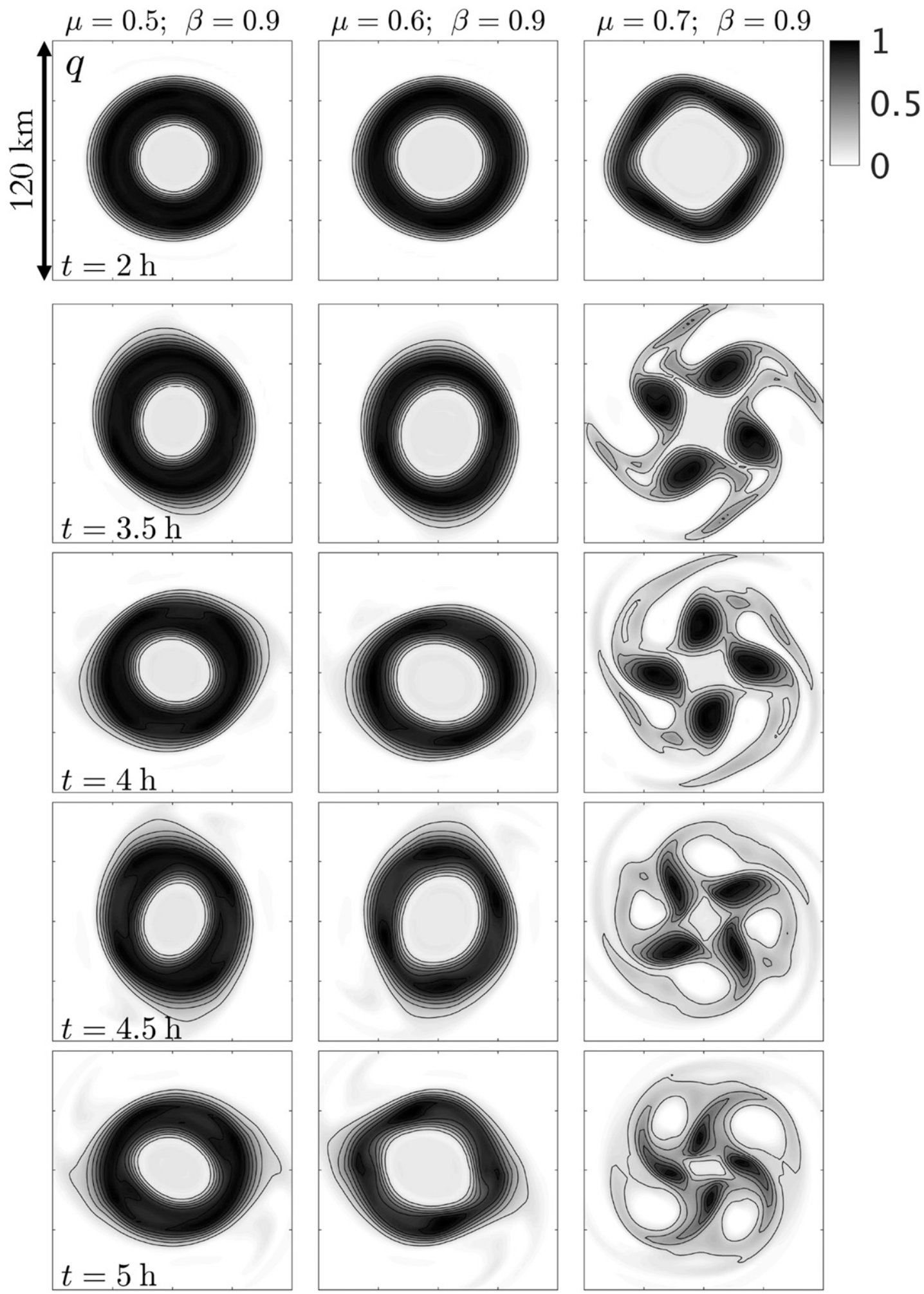

FIG. 15. Third category of sensitivity experiments. Snapshots at (from top to bottom) $t=2,3.5,4,4.5,5 \mathrm{~h}$ of the dry isentropic potential vorticity $q$ at $\theta=367 \mathrm{~K}$. The vortex parameters are (left) $\mu=0.5, \beta=0.9$; (middle) $\mu=0.6, \beta=0.9$; and (right) $\mu=0.7, \beta=0.9$. In any given panel, the plotted values are normalized to the given maximum value of $q$. 
Figure 15 shows snapshots of total PV over time for the three cases, which here will be referred to as wide (left column: $\mu=0.5$ ), intermediate (middle column: $\mu=0.6$ ), and thin annulus (right column: $\mu=0.7$ ). Regarding the wide annulus, it can be seen that after $2 \mathrm{~h}$ of simulation, the vortex is nearly symmetric. By $3.5 \mathrm{~h}$, the core has already attained a slight elliptical shape. After $5 \mathrm{~h}$, the core is still elliptical with a more pronounced and more intense (not shown) deformation. Upon careful inspection, one may detect that the orientation of the ellipse at the inner edge of the vortex differs from the orientation of the ellipse on the outer edge. This structure may serve as a hint (in a more qualitative sense) of the presence of two phase-shifted perturbations (in this case the two VR waves). Moving along to the intermediate annulus, the early evolution of the vortex is similar to the wide annulus in that the core remains nearly symmetric after $2 \mathrm{~h}$ and it becomes elliptical by $3.5 \mathrm{~h}$. On the other hand, after $4.5 \mathrm{~h}$, the two cases exhibit some early differences. Here and in contrast to the wide annulus, the inner edge of the core appears to have more of a square shape (instead of elliptical). This change in pattern indicates a shift of the fastest-growing core asymmetry from $n=2$ to one characterized by $n=4$. By $5 \mathrm{~h}$, the square deformation has become more discernible. Of interest to note is that despite the new polygonal shape, the outer edge of the vortex maintains a near-elliptical form. Overall, the evolution of the core deformation is envisioned to be the outcome of an early spontaneous radiative imbalance boosted by barotropic instability. In time and as the vortex core deformation amplifies, barotropic instability surpasses radiative instability to become the dominant destabilizing mechanism that deformed the core into a polygonal shape. Switching to the thin annulus, the results are quite different. Unlike the aforementioned two cases, by $2 \mathrm{~h}$, the vortex core has already deformed into an $n=4$ polygonal shape. Soon after $(<2 \mathrm{~h})$, the vortex breaks down into four distinct mesovortices. This is a clear example where barotropic instability is the fastest destabilizing mechanism, and spontaneous imbalance becomes of secondary importance.

\section{Concluding remarks}

Observations often reveal the existence of distinct asymmetries within the cores of intense tropical cyclones (TCs). Evidence suggests that these asymmetries may strongly modulate the storm characteristics and even their intensity. Despite this, considerable efforts to understand the physics and dynamics of TCs has been attempted using theory and models that assume axial symmetry. As a result, fundamental questions remain about the origin and role of asymmetries.
By TC asymmetries, we refer to vortex misalignments, elliptical eyewalls, and triangular and polygonal shapes. In this study, we revisit the potential mechanisms behind one of the most frequently observed: the elliptical eyewalls. Previously, it was suggested that elliptical eyewalls primarily result from barotropic instability, which may operate at different regions of the vortex: (i) across an annular eyewall because of the coupling and mutual growth of two vortex Rossby (VR) waves excited on the two eyewall edges (Reasor et al. 2000) and (ii) in the case of a concentric eyewall pattern, because of the interaction of the VR waves excited on the outer edge of the inner eyewall and the inner edge of the outer eyewall (Kossin et al. 2000; Oda et al. 2005).

The aforementioned results were largely based on simple models that filtered out inertia-gravity (IG) waves. Incorporating IG waves into the system introduces the possibility of an additional instability, the spontaneous radiative imbalance. This mechanism involves a VR wave in the TC core that spontaneously emits a frequency-matched spiral IG wave into the environment. Here, we argued that elliptical eyewalls, which may form within more realistic numerical models, can originate solely because of spontaneous imbalance.

Our control experiment is implemented in a dry threedimensional nonlinear primitive-equation model initialized with a balanced mature nonconvective annular TC-like vortex. We presented evidence that in this system, which supports both barotropic and radiative instability, the resultant amplifying elliptical deformation was caused by one azimuthal wavenumber $(n=2)$ baroclinic VR wave (excited on the outer edge of the vortex annulus) that spontaneously amplified as a result of producing radiation. No sign of a second VR wave (excited on the inner edge) was found, thereby ruling out the possibility of barotropic instability. The amplification of the ellipticity was explained based on the conservation of angular pseudomomentum. Primarily, the VR wave is associated with positive pseudomomentum. On the other hand, the spiral IG wave was shown to have negative pseudomomentum that amplifies with time. Thus, producing IG wave radiation compels the VR wave to grow. Our arguments were further supported by an independent linear eigenmode analysis and a linear simulation. It was shown that the dominant asymmetry coming out from the nonlinear model closely resembles the most unstable radiative linear eigenmode. The evolution of the asymmetry appeared to be in good agreement with a linear integration in which spontaneous imbalance dominates.

A number of sensitivity experiments performed on two pertinent shape parameters of the basic-state vortex (annulus thickness and hollowness) revealed that 
(i) spontaneous radiative imbalance can remain the dominant destabilizing mechanism for various vortices and thus provides confidence that the main findings are not restricted to a particular setup and (ii) the deformation of vortex core can sometimes be the result of a multimechanistic process where both radiative imbalance and barotropic instability operate simultaneously. We did not attempt to quantitatively assess the role of each mechanism because without the right diagnostic this task can lead to inconsistencies (Schecter and Menelaou 2017). We plan to report on this in a future paper. In closing, we wish to note that the idealized experiments described herein exclude both deep convection and boundary layer processes. Thus, they are an oversimplification of real TCs. The broader applicability of the present findings to actual TCs requires further investigation using in part high-resolution cloud-resolving simulations.

Acknowledgments. The authors thank three anonymous reviewers for their constructive comments. This research was sponsored by the Natural Science and Engineering Research Council of Canada and Hydro-Quebec through the IRC program. Konstantinos Menelaou would like to thank his postdoctoral mentor Dr. David Schecter for stimulating discussions and insightful comments. The simulation data used in this paper can be obtained from the first author, upon request. Konstantinos Menelaou dedicates this work to his son Menelaos for being the major source of his inspiration and motivation.

\section{APPENDIX}

\section{Empirical Normal Mode Analysis}

To extract the dominant wave patterns from the dataset, the empirical normal mode (ENM) technique developed by Brunet (1994) is used. This method has statistical properties similar to empirical orthogonal functions (EOFs) and dynamical properties of normal modes. In fact, the architecture of the two methods is similar (both are eigenvalue problems), and their only main difference is that EOFs are based on the statistical notion of variance whereas ENMs make use of a global invariant, the wave activity. Briefly, consider $\mathbf{P}^{\prime}$ being a state vector, a multicomponent field that may include, for example, velocity and density perturbations. This vector can be decomposed as

$$
\mathbf{P}^{\prime}(t, \mathbf{s})=\sum_{l=1}^{L} \boldsymbol{\alpha}_{l}(t) \mathbf{P}_{l}^{\prime}(\mathbf{s})+\epsilon(t, \mathbf{s}),
$$

where $t$ and $\mathbf{s}$ denote time and space, respectively, $L$ is the number of modes contained in the field, $\mathbf{P}_{l}^{\prime}(\mathbf{s})$ is a set of basis functions of space, $\boldsymbol{\alpha}_{l}(t)$ is a set of expansion functions of time, and $\epsilon$ is the decomposition error. The functions $\mathbf{P}_{l}^{\prime}(\mathbf{s})$ and $\boldsymbol{\alpha}_{l}(t)$ satisfy the following biorthogonality conditions:

$$
\begin{aligned}
\int d \mathbf{s} \mathbf{P}_{k}^{\prime \mathrm{T}}(\mathbf{s}) \mathbf{M}(\mathbf{s}) \mathbf{P}_{l}^{\prime}(\mathbf{s}) & =\lambda_{k} \delta_{k l}, \\
\overline{\boldsymbol{\alpha}_{k}(t) \boldsymbol{\alpha}_{l}(t)} & =\delta_{k l},
\end{aligned}
$$

Here, the overbar denotes time average, the superscript T indicates transposition, $\delta_{k l}$ is the Kronecker delta, and $\lambda_{k}$ is the square norm of the $k$ th basis function. By choosing the basis functions to minimize the mean square error, the following eigenvalue problem results:

$$
\mathbf{M} \mathbf{P}_{l}^{\prime}=\lambda_{l} \mathbf{P}_{l}^{\prime},
$$

in which

$$
\mathbf{M} \mathbf{P}_{l}^{\prime} \equiv \int d \mathbf{s}_{2} \mathbf{C}\left(\mathbf{s}_{1}, \mathbf{s}_{2}\right) \mathbf{M}\left(\mathbf{s}_{2}\right) \mathbf{P}_{l}^{\prime}\left(\mathbf{s}_{2}\right),
$$

and

$$
\mathbf{C}\left(\mathbf{s}_{1}, \mathbf{s}_{2}\right)=\overline{\mathbf{P}^{\prime}\left(t, \mathbf{s}_{1}\right) \mathbf{P}^{\prime T}\left(t, \mathbf{s}_{2}\right)} .
$$

Above, $\mathbf{C}$ is the space covariance matrix.

Note that to this point the metric $\mathbf{M}$ remains unspecified. Considering the Euclidean metric $\mathbf{M}=1$ leads to the standard EOF method, where the eigenvector of Eq. (A4) $\mathbf{P}_{l}^{\prime}$ is an EOF and the eigenvalue $\lambda_{l}$ measures the variance captured by $\mathbf{P}_{l}^{\prime}$. The variable $\boldsymbol{\alpha}_{l}$ is the corresponding principal component (PC). The ENM method uses a different metric derived from the conservation laws of linearized dynamics. To elaborate, consider a quadratic wave activity $\mathcal{H}$, which is globally conserved in the absence of any sources and sinks, that is,

$$
\frac{d \mathcal{H}}{d t}=0
$$

where

$$
\mathcal{H}=\int d \mathbf{s} \mathbf{P}_{k}^{\prime \mathrm{T}}(\mathbf{s}) \mathbf{M}_{\mathcal{H}}(\mathbf{s}) \mathbf{P}_{l}^{\prime}(\mathbf{s})
$$

It can be shown that the normal modes of the linearized system are orthogonal with respect to $\mathbf{M}_{\mathcal{H}}$ and that the wave activity $\mathcal{H}$ is a sum of modal contributions (Held 1985). If instead of the Euclidean norm we choose the wave activity metric $\mathbf{M}_{\mathcal{H}}, \mathbf{P}_{l}^{\prime}$ is called an ENM, $\lambda_{l}$ now represents the amount of wave activity carried by an ENM, and $\boldsymbol{\alpha}_{l}$ is still named a PC. 

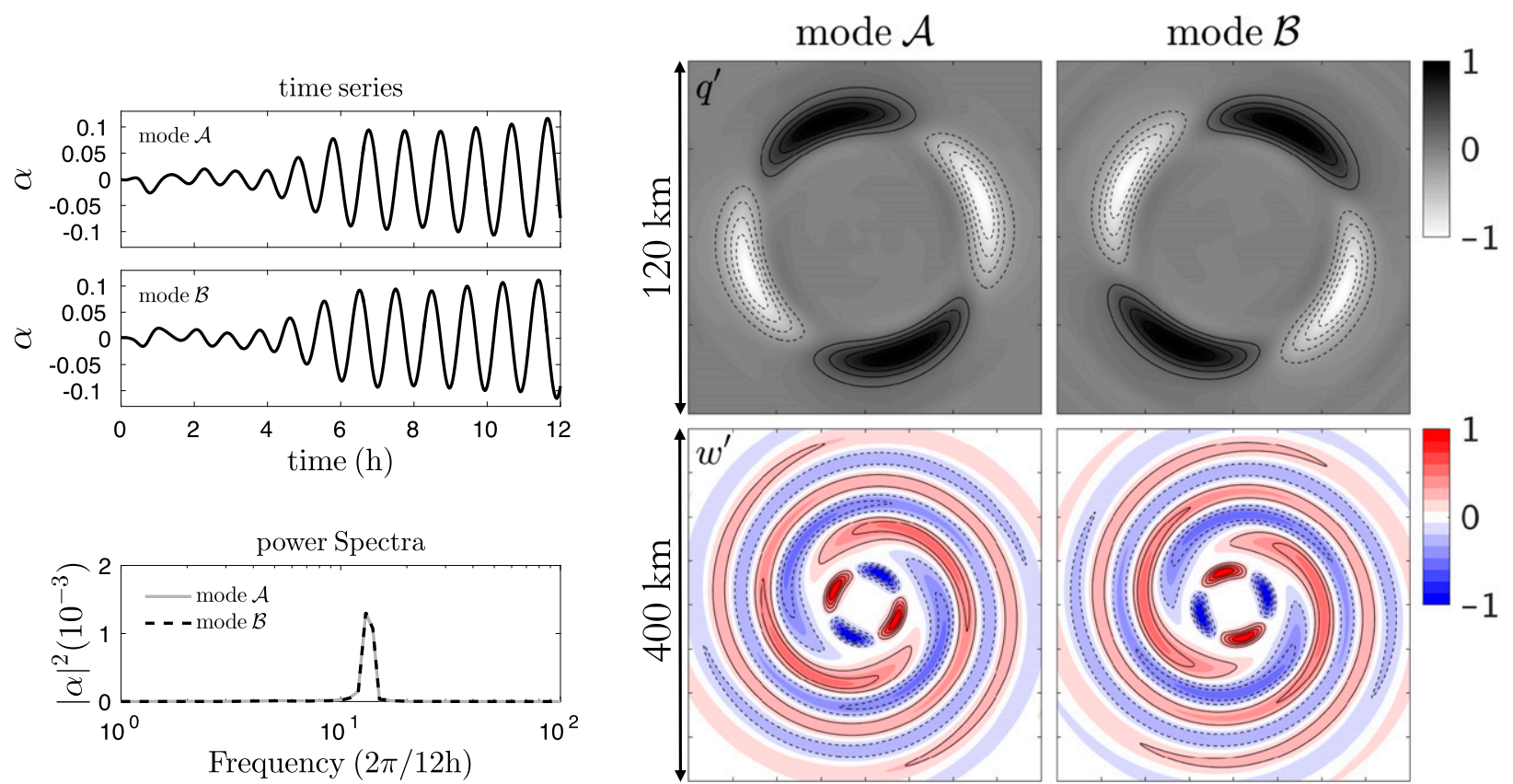

FIG. A1. (left) Time series and power spectra for ENMs $\mathcal{A}$ and $\mathcal{B}$. (center),(right) Contour plots of (top) $q^{\prime}$ at $\theta=303 \mathrm{~K}$ and (bottom) $w^{\prime}$ at $\theta=307 \mathrm{~K}$ associated with the $n=2$ dominant ENMs $\mathcal{A}$ and $\mathcal{B}$. The contour plots are normalized to the maximum value of the given perturbation field.

To apply the ENM method, we need to find $\mathbf{M}_{\mathcal{H}}$. For our particular application and for consistency with the main text, we choose to work with angular pseudomomentum. As a reminder, the angular pseudomomentum density $\mathcal{D}$ is defined as

$$
\mathcal{D}=-r v^{\prime} \sigma^{\prime}-\frac{r \bar{\sigma}^{2}\left(q^{\prime}\right)^{2}}{2 \partial \bar{q} / \partial r}
$$

The symbols used on the right-hand side of Eq. (A9) have the same meaning as in the main text. If we define the state vector $\mathbf{P}^{\prime}$ to be

$$
\mathbf{P}^{\prime} \equiv\left[v^{\prime}, \sigma^{\prime}, q^{\prime}\right]^{\mathrm{T}}
$$

we seek a matrix $\mathbf{M}_{\mathcal{H}}$ so that $\mathcal{D}$ can be written as

$$
\mathcal{D}=\mathbf{P}^{\prime \mathrm{T}} \mathbf{M}_{\mathcal{H}} \mathbf{P}^{\prime}
$$

which is

$$
\mathbf{M}_{\mathcal{H}}=-\frac{r}{2}\left(\begin{array}{ccc}
0 & 1 & 0 \\
1 & 0 & 0 \\
0 & 0 & \frac{\bar{\sigma}^{2}}{\partial \bar{q} / \partial r}
\end{array}\right) \text {. }
$$

From a practical aspect, to build ENMs using the control WRF simulation, we first interpolate the model data onto an isentropic coordinate system centered at the vortex core. Then we define the perturbation fields to be deviations from an azimuthal invariant basic state. After, the fields are decomposed as a sum of Fourier modes in the azimuthal direction. We will consider only azimuthal wavenumber- 2 perturbations since they are the most pertinent to our experiment.

Before proceeding, we point out that one ENM can only act as a standing wave. To form a propagating wave, we seek at least two modes with similar contribution to the total variance (degenerate eigenvalues), that have the same oscillation frequency, and they are in quadrature $\left(90^{\circ}\right.$ phase difference). Figure A1 illustrates the dominant pair of ENMs (in terms of PV and vertical velocity) and their corresponding time series (PCs) and power spectra. This pair of modes contribute $78.5 \%$ (mode $\mathcal{A}: 39.7 \%$, and mode $\mathcal{B}: 38.8 \%$ ) to the total variance of the wavenumber- 2 perturbation. Here, variance is defined as the ratio of the absolute value of the pseudomomentum for one mode and the total absolute value of the pseudomomentum for one specified wavenumber. The power spectra indicate that both modes have the same peak frequency and the orientation of their spatial patterns that they are in quadrature. In addition, from inspecting the time series, it is seen that at approximately $4 \mathrm{~h}$, the two modes begin to amplify. Taken together, the dominant pattern is suggestive of a propagating wave that amplifies with time. This wave 
closely resembles (both in terms of PV and vertical velocity) the perturbation patterns illustrated in Fig. 3 of the main text, verifying that the spontaneous radiative grow of the outer-edge VR wave is the dominant signal within the nonlinear WRF control simulation.

\section{REFERENCES}

Aberson, S. D., J. P. Dunion, and F. D. Marks Jr., 2006: A photograph of a wavenumber-2 asymmetry in the eye of Hurricane Erin. J. Atmos. Sci., 63, 387-391, https://doi.org/10.1175/ JAS3593.1.

Billant, P., and S. Le Dizès, 2009: Waves on a columnar vortex in a strongly stratified fluid. Phys. Fluids, 21, 106602, https://doi.org/ 10.1063/1.3248366

Braun, S. A., 2002: A cloud-resolving simulation of Hurricane Bob (1991): Storm structure and eyewall buoyancy. Mon. Wea. Rev. 130, 1573-1592, https://doi.org/10.1175/1520-0493(2002)130<1573: ACRSOH $>2.0 . \mathrm{CO} ; 2$.

Briggs, R. J., J. D. Daugherty, and R. H. Levy, 1970: Role of Landau damping in crossed-field electron beams and inviscid shear flow. Phys. Fluids, 13, 421-432, https://doi.org/10.1063/ 1.1692936.

Brunet, G., 1994: Empirical normal-mode analysis of atmospheric data. J. Atmos. Sci., 51, 932-952, https://doi.org/10.1175/1520-0469(1994) 051<0932:ENMAOA $>2.0 . C O ; 2$.

Chen, Y., G. Brunet, and M. K. Yau, 2003: Spiral bands in a simulated hurricane. Part II: Wave activity diagnostics. J. Atmos. Sci., 60, 1239-1256, https://doi.org/10.1175/1520-0469(2003) $60<1239$ :SBIASH $>2.0$. CO 2 .

Corbosiero, K. L., J. Molinari, A. R. Aiyyer, and M. L. Black, 2006 The structure and evolution of Hurricane Elena (1985). Part II: Convective asymmetries and evidence for vortex Rossby waves. Mon. Wea. Rev., 134, 3073-3091, https://doi.org/ 10.1175/MWR3250.1.

Ford, R., 1994a: The instability of an axisymmetric vortex with monotonic potential vorticity in rotating shallow water. J. Fluid Mech., 280, 303-334, https://doi.org/ 10.1017/S0022112094002946.

_ 1994b: The response of a rotating ellipse of uniform potential vorticity to gravity wave radiation. Phys. Fluids, 6, 3694-3704, https://doi.org/10.1063/1.868360.

Held, I. M., 1985: Pseudomomentum and the orthogonality of modes in shear flows. J. Atmos. Sci., 42, 2280-2288, https://doi.org/ 10.1175/1520-0469(1985)042<2280:PATOOM>2.0.CO;2.

Hodyss, D., and D. S. Nolan, 2008: The Rossby-inertia-buoyancy instability in baroclinic vortices. Phys. Fluids, 20, 096602 , https://doi.org/10.1063/1.2980354.

Hoskins, B. J., and F. P. Bretherton, 1972: Atmospheric frontogenesis models: Mathematical formulation and solution. J. Atmos. Sci., 29, 11-37, https://doi.org/10.1175/1520-0469(1972)029<0011 AFMMFA $>2.0 . \mathrm{CO} ; 2$.

Jordan, C. L., 1958: Mean soundings for the West Indies area. J. Meteor., 15, 91-97, https://doi.org/10.1175/1520-0469(1958) 015<0091:MSFTWI>2.0.CO;2.

Kossin, J. P., W. H. Schubert, and M. T. Montgomery, 2000: Unstable interactions between a hurricane's primary eyewall and a secondary ring of enhanced vorticity. J. Atmos. Sci., 57, 3893-3917, https://doi.org/10.1175/1520-0469(2001)058<3893: UIBAHS $>2.0 . \mathrm{CO} ; 2$.

Kuo, H.-C., R. T. Williams, and J.-H. Chen, 1999: A possible mechanism for the eye rotation of Typhoon Herb. J. Atmos.
Sci., 56, 1659-1673, https://doi.org/10.1175/1520-0469(1999) 056<1659:APMFTE $>2.0 . C O ; 2$.

Li, X., J. A. Zhang, X. Yang, W. G. Pichel, M. DeMaria, D. Long, and Z. Li, 2013: Tropical cyclone morphology from spaceborne synthetic aperture radar. Bull. Amer. Meteor. Soc., 94, 215-230, https://doi.org/10.1175/BAMS-D-11-00211.1.

Menelaou, K., D. A. Schecter, and M. K. Yau, 2016: On the relative contribution of inertia-gravity wave radiation to asymmetric instabilities in tropical cyclone-like vortices. J. Atmos. Sci., 73, 3345-3370, https://doi.org/10.1175/JAS-D15-0360.1; Corrigendum, 73, 4605, https://doi.org/10.1175/ JAS-D-16-0261.1.

Mitsuta, Y., and S. Yoshizumi, 1973: Periodic variations of pressure, wind and rainfall observed at Miyakojima during the second Miyakojima typhoon. J. Meteor. Soc. Japan, 51, 475485, https://doi.org/10.2151/jmsj1965.51.6_475.

Naylor, J., and D. A. Schecter, 2014: Evaluation of the impact of moist convection on the development of asymmetric inner core instabilities in simulated tropical cyclones. J. Adv. Model. Earth Syst., 6, 1027-1048, https://doi.org/10.1002/ 2014MS000366.

Nolan, D. S., M. T. Montgomery, and L. D. Grasso, 2001: The wavenumber-one instability and trochoidal motion of hurricanelike vortices. J. Atmos. Sci., 58, 3243-3270, https://doi.org/ 10.1175/1520-0469(2001)058<3243:TWOIAT $>2.0$. CO; 2 .

Nuissier, O., R. F. Rogers, and F. Roux, 2005: A numerical simulation of Hurricane Bret on 22-23 August 1999 initialized with airborne Doppler radar and dropsonde data. Quart. J. Roy. Meteor. Soc., 131, 155-194, https://doi.org/10.1256/ qj.02.233.

Oda, M., T. Itano, G. Naito, M. Nakanishi, and K. Tomine, 2005: Destabilization of the symmetric vortex and formation of the elliptical eye of Typhoon Herb. J. Atmos. Sci., 62, 2965-2976, https://doi.org/10.1175/JAS3521.1.

Park, J., and P. Billant, 2013: Instabilities and waves on a columnar vortex in a strongly stratified and rotating fluid. Phys. Fluids, 25, 086601, https://doi.org/10.1063/1.4816512.

Plougonven, R., and V. Zeitlin, 2002: Internal gravity wave emission from a pancake vortex: An example of wave-vortex interaction in strongly stratified flows. Phys. Fluids, 14, 1259-1268, https:// doi.org/10.1063/1.1448297.

Reasor, P. D., M. T. Montgomery, F. D. Marks Jr., and J. F. Gamache, 2000: Low-wavenumber structure and evolution of the hurricane inner core observed by airborne dual-Doppler radar. Mon. Wea. Rev., 128, 1653-1680, https://doi.org/10.1175/1520-0493(2000) $128<1653$ :LWSAEO $>2.0$. CO 2 .

Schecter, D. A., 2008: The spontaneous imbalance of an atmospheric vortex at high Rossby number. J. Atmos. Sci., 65, 2498-2521, https://doi.org/10.1175/2007JAS2490.1.

2015: Response of a simulated hurricane to misalignment forcing compared to the predictions of a simple theory. J. Atmos. Sci., 72, 1235-1260, https://doi.org/10.1175/JAS-D-14-0149.1.

- , and M. T. Montgomery, 2003: On the symmetrization rate of an intense geophysical vortex. Dyn. Atmos. Oceans, 37, 55-88, https://doi.org/10.1016/S0377-0265(03)00015-0.

— , and — 2004: Damping and pumping of a vortex Rossby wave in a monotonic cyclone: Critical layer stirring versus inertia-buoyancy wave emission. Phys. Fluids, 16, 1334-1348, https://doi.org/10.1063/1.1651485.

, and - 2006: Conditions that inhibit the spontaneous radiation of spiral inertia-gravity waves from an intense mesoscale cyclone. J. Atmos. Sci., 63, 435-456, https://doi.org/ 10.1175/JAS3641.1. 
, and - 2007: Waves in a cloudy vortex. J. Atmos. Sci., 64, 314-337, https://doi.org/10.1175/JAS3849.1.

, and K. Menelaou, 2017: Note on analyzing perturbation growth in a tropical cyclone-like vortex radiating inertia-gravity waves. J. Atmos. Sci., 74, 1561-1571, https://doi.org/10.1175/ JAS-D-16-0289.1.

Schubert, W. H., M. T. Montgomery, R. K. Taft, T. A. Guinn, S. R. Fulton, J. P. Kossin, and J. P. Edwards, 1999: Polygonal eyewalls, asymmetric eye contraction, and potential vorticity mixing in hurricanes. J. Atmos. Sci., 56, 1197-1223, https://doi.org/ 10.1175/1520-0469(1999)056<1197:PEAECA > 2.0.CO;2.

Shapiro, L. J., and M. T. Montgomery, 1993: A three-dimensional balance theory for rapidly rotating vortices. J. Atmos. Sci., 50,
3322-3335, https://doi.org/10.1175/1520-0469(1993)050<3322: ATDBTF $>2.0 . \mathrm{CO} ; 2$.

Skamarock, W. C., and Coauthors, 2008: A description of the Advanced Research WRF version 3. NCAR Tech. Note NCAR/TN-475+STR, 113 pp., https://doi.org/10.5065/ D68S4MVH.

Takehiro, S.-I., and Y.-Y. Hayashi, 1992: Over-reflection and shear instability in a shallow-water model. J. Fluid Mech., 236, 259-279, https://doi.org/10.1017/S0022112092001411.

Wang, Y., and C.-C. Wu, 2004: Current understanding of tropical cyclone structure and intensity changes-A review. Meteor. Atmos. Phys., 87, 257-278, https://doi.org/10.1007/ s00703-003-0055-6. 